\title{
Neural crest cells regulate optic cup morphogenesis by promoting extracellular matrix assembly
}

Chase D. Bryan ${ }^{1}$, Rebecca L. Pfeiffer ${ }^{2}$, Bryan W. Jones ${ }^{2}$, Kristen M. Kwan ${ }^{1, *}$

\section{Affiliations}

${ }^{1}$ Department of Human Genetics, University of Utah, Salt Lake City, UT, USA

${ }^{2}$ Department of Ophthalmology and Visual Sciences, John A. Moran Eye Center, University of Utah School of Medicine, Salt Lake City, UT, USA

* Correspondence to: Department of Human Genetics, EIHG 5100, University of Utah Medical Center, 15 North 2030 East, Salt Lake City, UT 84112, USA.

Email address: kmkwan@genetics.utah.edu 


\section{Abstract}

2 The interactions between an organ and its surrounding environment are critical in regulating its

3 development. In vertebrates, neural crest and mesodermal mesenchymal cells have been

4 observed close to the eye during development, and mutations affecting this periocular

5 mesenchyme can cause defects in early eye development, yet the underlying mechanism has

6 been unknown. Here, using timelapse microscopy and four-dimensional cell tracking in

7 zebrafish, we establish that genetic loss of neural crest impairs cell movements within the optic

8 vesicle. At the ultrastructural level, neural crest cells are required for basement membrane

9 formation specifically around the retinal pigment epithelium. Neural crest cells express the

10 extracellular matrix crosslinking protein nidogen and, strikingly, ectopically expressing nidogen

11 in the absence of neural crest partially restores optic cup morphogenesis. These results

12 demonstrate that the neural crest is required for local establishment of ocular extracellular matrix

13 superstructure, which in turn drives optic cup morphogenesis.

\section{Introduction}

Vertebrate eye development begins with specification of the eye field, followed by a

17 series of tissue movements that together comprise optic cup morphogenesis (Hilfer, 1983;

18 Schmitt and Dowling, 1994; Schook, 1980; Walls, 1942). Initially, a pair of optic vesicles

19 evaginate bilaterally from the developing forebrain; the bilayered optic vesicles give rise to the

20 neural retina and retinal pigment epithelium (RPE). As these vesicles elongate, the connection

21 between the vesicle and brain is constricted, generating the optic stalk. Multiple cell and tissue

22 movements occur during invagination, the final stage of optic cup morphogenesis: the optic

23 vesicles buckle and take on a hemispherical shape to enwrap the lens as it invaginates from the 
24 overlying ectoderm. Additionally, the optic fissure forms along the ventral side of the optic

25 vesicle and optic stalk. Lineage tracing and live imaging experiments performed in zebrafish

26 have enabled cellular-level analysis of the morphogenetic movements that occur during these

27 stages of eye development. These experiments have revealed that during invagination, a subset

28 of cells that arise on the medial layer of the optic vesicle migrate around the rim of the vesicle

29 and eventually take up residence within the lateral layer, contributing to the neural retina; those

30 which remain on the medial layer flatten and comprise the RPE (Heermann et al., 2015; Kwan et

31 al., 2012; Li et al., 2000; Picker et al., 2009; Sidhaye and Norden, 2017). Despite

32 characterization of these cellular movements, the cellular and molecular mechanisms underlying

33 many aspects of these processes are not well understood.

In addition to these complex tissue movements and rearrangements, the optic vesicle undergoes morphogenesis in a complex environment containing multiple extraocular tissues,

36 including the overlying ectoderm from which the lens will develop, the prospective brain, and

37 the periocular mesenchyme (POM). Mesenchymal cells influence the development and

38 morphogenesis of many epithelial organs, such as tooth and salivary gland, by producing growth

39 factors and signaling molecules such as BMPs, WNTs, and FGFs (Thesleff, 2003; Wells et al.,

40 2013), or through modifications to the extracellular matrix (ECM) that surrounds developing

41 epithelia. For example, mesenchymal cells can influence morphogenesis through cleavage and

42 destruction of ECM via proteolysis: in the developing mouse lung, mesenchymally-expressed

43 matrix metalloproteinase-2 (MMP-2) is required for branching morphogenesis (Kheradmand et

44 al., 2002). Conversely, mesenchymal cells can also promote epithelial morphogenesis through

45 deposition of new ECM components such as laminin and nidogen, reviewed in (Nelson and 
Previous work has indicated a role for epithelial-mesenchymal interactions during optic cup morphogenesis, although the exact role and molecular nature of these interactions are poorly understood. The POM is a heterogeneous cell population in close proximity to the optic cup, comprised of neural crest cells and mesodermally-derived mesenchyme (Johnston et al., 1979),

51 and multiple tissues in the mature eye are derived in part from these mesenchymal cell

52 populations (Williams and Bohnsack, 2015). Recent work also indicates a role for POM in

53 closure of the optic fissure, a developmental step subsequent to optic cup formation (Gestri et al.,

54 2018). Yet in addition to these later roles, disruptions to transcription factors expressed in the

55 POM during early eye development, such as ap2a, pitx2 or zic2, lead to severe optic cup

56 malformations (Bassett et al., 2010; Bohnsack et al., 2012; Gestri et al., 2009; Li and Cornell,

57 2007; Sedykh et al., 2017). These data suggest a critical role for the POM in regulating optic cup

58 morphogenesis, possibly through regulating direct or indirect signaling to the optic cup.

59 Communication between these tissues appears to be bidirectional: the developing optic vesicle is known to signal to the migratory neural crest, in part through retinoic acid and PDGF signaling

61 (Bohnsack et al., 2012; Eberhart et al., 2008; Lupo et al., 2011). In zebrafish rx3 mutants, eye specification fails to occur; these embryos subsequently display aberrant craniofacial neural crest

63 migration (Langenberg et al., 2008), indicating that the eye is at least partially responsible for

64 facilitating proper neural crest migration. However, many of the molecules that mediate these

65 bidirectional epithelial-mesenchymal interactions during optic cup morphogenesis have yet to be 66 discovered.

Although there are hints that secreted morphogens such as Hedgehog or TGF- $\beta$ may be crucial for crosstalk between the optic vesicle and the POM (Fuhrmann et al., 2000; Grocott et 
modifying the ECM. A complex ECM has long been known to surround the developing optic vesicle throughout optic cup morphogenesis (Dong and Chung, 1991; Hendrix and Zwaan, 1975; Kwan, 2014; Peterson et al., 1995; Svoboda and O'Shea, 1987; Tuckett and Morriss-Kay, 1986), yet the dynamics of ECM deposition and remodeling around the developing eye are poorly understood. Only recently have specific roles of fibronectin (Hayes et al., 2012; Huang et al., 2011) and laminin (Bryan et al., 2016; Ivanovitch et al., 2013; Sidhaye and Norden, 2017) during optic cup morphogenesis been elucidated, and both of these ECM proteins are expressed by the optic vesicle itself. POM cells could modify the ocular ECM by expressing ECM-degrading proteins such as metalloproteases, or by providing structural ECM proteins such as nidogens: both are expressed by mesenchymal cells during morphogenesis of other organs. The roles of many ECM proteins during optic cup morphogenesis, especially those which may be produced by mesenchymal cells, have not been studied in detail, and their functions during this particular process remain a mystery. The POM likely regulates multiple aspects of optic cup morphogenesis, yet many questions remain about the nature of the interactions between the POM and the developing eye. In this study, we sought to determine the role of the neural crest in regulating morphogenesis of the developing optic cup. When and where do neural crest cells interact with the optic vesicle during optic cup morphogenesis? Is the neural crest cell population actually required for optic cup morphogenesis? Are there morphogenetic events within the developing eye that depend on the neural crest? At a molecular level, how do neural crest cells interact with and regulate behaviors within the optic cup? Here we demonstrate that loss of neural crest cells, via two

91 independent genetic methods, impairs optic cup morphogenesis. Using 4-dimensional timelapse 2 imaging and computational methods, we pinpoint specific cell movements within the optic cup 
93 that are dependent on the neural crest. We further demonstrate that loss of neural crest leads to a

94 dramatic disruption of basement membrane formation, but only around the RPE. Finally, we

95 uncover a key molecular effector of neural crest crosstalk with the eye: our results indicate that

96 neural crest cells regulate optic cup morphogenesis through deposition of nidogens, crucial

97 modulators of ECM structure. 
98

99

100

101

102

103

104

105

106

107

108

109

110

111

112

113

114

115

116

117

118

119

\section{Results}

Neural crest is in contact with the optic vesicle throughout optic cup morphogenesis

In zebrafish, optic cup morphogenesis occurs from 10-24 hours post fertilization (hpf), during which time the optic vesicles evaginate and undergo a series of stereotypical movements and shape changes to become the organized optic cup, comprised of the neural retina, retinal pigment epithelium (RPE), and lens. To begin to determine the nature of how the neural crest

cell population might affect optic cup morphogenesis, we first sought to identify when and where neural crest cells interact with developing eye tissues. To visualize both the developing eye and neural crest, we crossed two transgenic zebrafish lines: $\operatorname{Tg}(\text { bactin2:EGFP-CAAX })^{z 200}$, in which GFP ubiquitously labels cell membranes, and $\operatorname{Tg}(\operatorname{sox} 10: m e m R F P)^{v u 234}$ (Kirby et al., 2006), in which neural crest cell membranes are marked with membrane-bound RFP. Using embryos from this cross, we performed 4-dimensional timelapse imaging during optic cup morphogenesis, from 12.5 hpf-24.5 hpf (Fig 1, Movies S1, S2). At the start of our imaging at $12.5 \mathrm{hpf}$, neural crest cells are coming into contact with the posterior margin of the optic vesicle (Fig 1A, A'). Initially, neural crest cells migrate anteriorly in the space between the prospective brain and optic vesicle (Fig 1B, Movie S1); beginning around $16 \mathrm{hpf}$, the developing optic stalk is gradually enwrapped by neural crest cells (Fig 1B', Movie S2). Neural crest cells also migrate laterally and ventrally to encompass the posterior and ventral sides of the optic cup, appearing to be in close contact with the developing eye. By $24.5 \mathrm{hpf}$, the neural crest has entered the optic fissure and migrates toward the space between the neural retina and lens (Fig 1D', arrow). By the end of optic cup morphogenesis, neural crest-derived cells have encapsulated the RPE side of the optic cup (Fig 
$121 \quad$ Neural crest cells are required for proper optic cup morphogenesis

123 developing eye and the neural crest (Bohnsack et al., 2012; Eberhart et al., 2008; Grocott et al., 124 2011; Langenberg et al., 2008; Sedykh et al., 2017). Since we observed neural crest cells in 125 contact with the optic vesicles at early stages of eye development, we sought to determine

126 whether neural crest cells are required for proper optic cup morphogenesis. To test the

127 requirement for neural crest, we used two independent genetic models, both of which exhibit a 128 widespread depletion of neural crest.

130 of neural crest cells (Arduini et al., 2009; Wang et al., 2011). We crossed adult

131 tfap $2 a^{t s 213} ;$ foxd $3^{z d f 10}$ heterozygote carriers to two transgenic lines: $\operatorname{Tg}($ bactin2:EGFP-CAAX) and

$132 \operatorname{Tg}(\operatorname{sox} 10: m e m R F P)$. Crossing these two transgenic/double heterozygote lines enabled us to

133 visualize the optic cup as well as to assay the presence of any remaining neural crest cells in the

134 tfap2a;foxd3 double mutant. At 24 hpf, tfap2a;foxd3 double mutants display mild but

135 reproducible optic cup morphogenesis defects. At the dorsal-ventral midpoint of the optic cup, 136 the nasal side of the neural retina is flatter than in sibling control embryos and fails to completely

137 enwrap the lens, indicative of a defect in optic cup invagination (Fig 2A, B). Quantification of 138 optic cup invagination angle indicates a significant decrease in the extent to which the retina 139 enwraps the lens in tfap $2 a$;foxd 3 double mutants $\left(37.8 \pm 1.9^{\circ}\right)$ compared to wildtype controls $140\left(47.3 \pm 1.8^{\circ}\right.$; Fig $\left.2 \mathrm{G}\right)$. The optic fissure, a cleft-like structure along the ventral side of the optic 141 stalk and optic cup, is also aberrant in tfap $2 a ; f o x d 3$ double mutants. At 24 hpf, control embryos 142 display two closely apposed fissure margins (Fig $2 \mathrm{H}$ ) while the margins in tfap2a;foxd3 double 143 mutants are wider set, indicating that optic fissure development is abnormal (Fig 2I). Visualizing 
144 sox 10:memRFP-positive cells indicates that, as expected, neural crest cells are substantially

145 reduced in the vicinity of the optic cup in tfap $2 a$;foxd3 double mutants (Fig $2 \mathrm{E}, \mathrm{L}$ ) compared to

146 wildtype controls (Fig 2D, K), with a notable absence on the nasal side of the optic cup (Fig 2L).

147 Since incrosses of tfap2a;foxd3 heterozygote adults yield both single as well as double mutant

148 genotypes, we characterized tfap $2 a$ and foxd 3 single mutants as well. Neither single mutant

149 displays as apparent optic cup morphogenesis defects or decrease in invagination angle as the

150 double mutant (Fig S1A, B, E), likely due to the presence of more neural crest cells in either

151 single mutant compared to the double mutant (Fig S1C, D).

As a second means of testing the requirement for neural crest cells in optic cup

morphogenesis, we assayed optic cup morphogenesis in alyron $^{z 24}$ (pafl) mutants in which neural

154 crest development is severely impaired (Cretekos and Grunwald, 1999). Although ubiquitously

155 expressed, disruptions to components of the RNA Polymerase II-Associated Factor (Paf1)

156 complex result in severe reductions in neural crest gene expression, coupled with developmental

157 defects in neural crest derived tissues (Akanuma et al., 2007; Langenbacher et al., 2011; Nguyen

158 et al., 2010). We observe that optic cup invagination is more severely disrupted in pafl mutants

$159\left(25.6 \pm 3.6^{\circ}\right)$ when compared to wildtype controls or tfap $2 a$;foxd3 double mutants (Fig $\left.2 \mathrm{C}, \mathrm{G}\right)$.

160 We also visualized neural crest in pafl mutants and saw a substantial reduction in

161 sox 10:memRFP-positive cells surrounding the optic cup at $24 \mathrm{hpf}(\mathrm{Fig} 2 \mathrm{~F}, \mathrm{M})$, similar to the

162 neural crest loss seen in the tfap2a;foxd3 double mutant. However, as paf1 and other members of

163 the Pafl complex are expressed ubiquitously (Nguyen et al., 2010; Thisse and Thisse, 2004), it is

164 possible that paf1 also plays an intrinsic role in development of the optic vesicle itself, which

165 could account for the more severe morphogenesis defects we observe in the pafl mutants. Thus, 
166 further analysis on the role of neural crest in optic cup morphogenesis was carried out solely

167 using the tfap $2 a$;foxd3 double mutant.

Previous studies have suggested a role for the periocular mesenchyme in closure of the

optic fissure along the ventral side of the retina and optic stalk (Gestri et al., 2018; Hero, 1990;

Hero et al., 1991; James et al., 2016; Lupo et al., 2011; Weiss et al., 2012). Consistent with these

171 data, we see optic fissure defects and gaps in ocular pigmentation (indicative of coloboma) in

$17259.38 \%$ of $t$ fap $2 a ; f o x d 3$ double mutants at $52 \mathrm{hpf}$ versus $7.62 \%$ of control embryos $(\mathrm{n}=19 / 32$ and

$173 \mathrm{n}=8 / 105$, respectively; data from three separate clutches; Fig 2N, O). However, these previous

174 studies have focused largely on later stages of optic fissure fusion, during which the POM appear

175 to play an active role. Therefore, our observations at $24 \mathrm{hpf}$ demonstrate that the neural crest

176 additionally plays a role in the early stages of optic cup morphogenesis.

$\underline{\text { TGF- }} \beta \underline{\text { signaling is unaffected by loss of neural crest, while Pax } 2 \mathrm{a} \text { expression is expanded }}$

The finding that neural crest cells are required for early stages of optic cup

morphogenesis raised the possibility that neural crest cells were providing a signaling cue to the

181 developing optic cup; an intriguing candidate we first tested was TGF- $\beta$ signaling. Work performed in chick optic vesicle explants demonstrated that POM cells are necessary for proper RPE specification and development, a requirement that could be bypassed with treatment with the TGF- $\beta$ family member Activin (Fuhrmann et al., 2000). Other experiments have suggested

185 that neural crest cells repress lens specification through TGF- $\beta$ signaling to ensure proper 186 positioning of the lens (Grocott et al., 2011). Thus, we sought to determine whether the neural 187 crest is necessary for TGF- $\beta$ signaling to the developing eye in zebrafish. Using an antibody 188 against phospho-Smad3 to detect sites of active TGF- $\beta$ signaling, we did not detect any 
189

190

191

192

193

194

195

196

197

198

199

200

201

202

203

204

205

206

207

208

209

210

211

differences in phospho-Smad3 localization between control and mutant optic cups at 24 hpf (Fig 3A, C vs 3B, D; Fig S2A-D). This result indicates that the neural crest subpopulation of POM is not required for proper TGF- $\beta$ signaling at the end of zebrafish optic cup morphogenesis.

As we observed morphogenetic abnormalities in nasal and ventral portions of the optic cup in tfap2a;foxd 3 double mutants, we hypothesized that the neural crest might be required for some aspect of optic cup patterning. Using an antibody against Pax2a, a transcription factor expressed in the ventral optic cup and optic stalk (Fig 3E, G), we found that Pax 2a expression is expanded into the RPE layer in tfap2a;foxd3 double mutants, in cells located more dorsally or temporally than observed in wildtype eyes (arrowheads in Fig 3F, H). We quantified the portion of the optic cup which expressed Pax2a as an angle (schematized in Fig 3J) and find that expression is significantly expanded in the tfap $2 a$; foxd 3 double mutant $\left(134.2 \pm 10.5^{\circ}\right)$ compared to control embryos $\left(100.3 \pm 5.9^{\circ}\right.$; Fig $\left.3 \mathrm{I}\right)$. This expansion was consistently observed in tfap $2 a ; f o x d 3$ double mutants as well as both tfap $2 a$ and foxd3 single mutants (Fig S2E-H). In other models of ventral optic cup mispatterning, especially due to aberrant or increased Hedgehog signaling, Pax2a is expanded not just into the RPE layer but throughout the ventral hemisphere of the retina (Lee et al., 2008; Sedykh et al., 2017). This phenotype we observe here is distinct in its restriction to the RPE layer, and suggests that perhaps gross mispatterning of the ventral optic cup does not occur when neural crest is lost, but rather, some aspect of cell movements within the optic cup is disrupted.

Neural crest cells are required for proper cell movements within the optic vesicle We observed optic cup invagination defects and ectopic Pax $2 \mathrm{a}$ expression in cells in the RPE layer at $24 \mathrm{hpf}$, which suggested that cell movements within the optic cup might be 
212 disrupted in the tfap2a;foxd3 double mutant. We therefore sought to pinpoint when and where

213 cell movements are disrupted in the tfap2a;foxd3 double mutant. Determining how widespread

214 movement defects are would provide clues regarding the nature and role of the interactions

215 between the optic vesicle and neural crest. We directed our attention to two movements executed

216 by cells which reside in the medial layer of the optic vesicle, as these are the cells potentially

217 interacting with the neural crest, as visualized in Figure 1. The first movement is that of

218 prospective RPE cells, while the second, rim involution, involves a subset of cells that migrate

219 around the developing optic cup rim from the medial layer into the lateral layer, the prospective

220 neural retina (Heermann et al., 2015; Kwan et al., 2012; Picker et al., 2009; Sidhaye and Norden,

221 2017). In mutant optic cups, expanded Pax2a expression in the RPE layer could result from

222 failure of Pax2a-expressing cells to undergo rim movement into the neural retina, thus remaining

223 in the apparent RPE layer of the optic cup; we hypothesized that rim involution might be

224 disrupted in the absence of neural crest cells. To test this possibility, as well as to determine how

225 widespread the cell movements are which depend on the neural crest, we performed live imaging

226 and 4-dimensional cell tracking of optic cup morphogenesis in wildtype and tfap2a;foxd3 double

227 mutant embryos (Fig 4, Movie S3).

As we saw distinct invagination defects in the nasal hemisphere of 24 hpf tfap $2 a$;foxd 3

229 double mutant optic cups, we began by selecting nasal RPE and retina cells in their final

230 positions at $24 \mathrm{hpf}$. We then tracked these cells retrospectively to establish their origins and

231 movements from optic vesicle stage; a subset of tracked cells and trajectories is shown in Figure

232 4. First we visualized RPE cell movements and found clear changes in the trajectory of

233 tfap2a;foxd3 double mutant cells. While the nasal RPE population arises in the same starting

234 position in both wildtype and double mutant embryos, wildtype nasal RPE cells execute a 
235 gradual, arc-like trajectory toward the nasal-lateral portion of the RPE and continually move

236 toward the anterior and lateral sides of the embryo (Fig 4C-C',', Movie S4). In contrast, the

237 tfap2a;foxd3 nasal RPE cells move in a straight, anterior direction until $19.5 \mathrm{hpf}$, when they

238 make a sudden, 90-degree turn in the lateral direction (Fig 4D-D',', Movie S5). To our surprise,

239 when we quantify these cell movements (Fig 4I-K), we find that nasal RPE cells move faster and

240 farther in tfap2a;foxd3 double mutants (average speed $=0.57 \pm 0.02 \mu \mathrm{m} / \mathrm{min}$; total distance $=$

$241385.75 \pm 15.71 \mu \mathrm{m}$ ) than wildtypes (average speed $=0.45 \pm 0.05 \mu \mathrm{m} / \mathrm{min}$; total distance $=$

$242303.01 \pm 34.06 \mu \mathrm{m}$; Fig 4I, J). Additionally, net displacement of nasal RPE cells is increased in

243 tfap2a;foxd3 double mutants $(170.76 \pm 14.55 \mu \mathrm{m})$ compared to wildtypes $(131.17 \pm 10.16 \mu \mathrm{m})$,

244 indicating that these cells do not arrive at the correct position within the optic cup. Taken

245 together, these data confirm that neural crest is required for proper migration and positioning of

246 nasal RPE cells during optic cup morphogenesis.

247 Through cell tracking, we also identified defects in the movements of nasal retinal cells.

248 We find that both wildtype (Fig 4E-E',', Movie S6) and tfap2a;foxd3 double mutant nasal retinal

249 cells (Fig 4F-F', ', Movie S7) arise in equivalent domains (Kwan et al., 2012) and follow a

250 similar trajectory during optic cup morphogenesis, with one key difference: while wildtype

251 retinal cells undergo a lateral turn away from the midline around $17.75 \mathrm{hpf}$ (Movie S6), that

252 same turn is delayed in the tfap2a;foxd3 mutant until approximately $19.5 \mathrm{hpf}$ (Movie S7).

253 Similar to the nasal RPE population, nasal retinal cells move faster and farther in the

254 tfap2a;foxd3 double mutant (average speed $=0.60 \pm 0.04 \mu \mathrm{m} / \mathrm{min}$; total distance $=402.80 \pm 17.33$

$255 \mu \mathrm{m}$ ) than wildtype (average speed $=0.53 \pm 0.03 \mu \mathrm{m} / \mathrm{min}$; total distance $=352.41 \pm 29.99 \mu \mathrm{m} ;$ Fig

$2564 \mathrm{I}, \mathrm{J}$ ), although net displacement of these cells is unchanged (wildtype $=111.55 \pm 18.18 \mu \mathrm{m}$, vs.

$257 \quad$ tfap $2 a ; f o x d 3=124.08 \pm 8.43 \mu \mathrm{m})$. 
morphological defects on the temporal side of the optic cup, we found differences between

264 the neural retina visible by $22 \mathrm{hpf}($ Fig 4G-G',', Movie S8). In the tfap2a;foxd3 double mutant,

265 these same cells do not begin migrating around the rim until 22 hpf (Fig 4 H-H', , Movie S9). As

266 observed with cells on the nasal side of the retina, temporal retinal cell speeds and distances

267 traveled are significantly increased in the tfap $2 a ;$ foxd 3 double mutant (average speed $=$

$0.57 \pm 0.04 \mu \mathrm{m} / \mathrm{min}$; total distance $=375.01 \pm 27.53 \mu \mathrm{m})$ compared to wildtype (average speed $=$ unchanged (wildtype $=78.50 \pm 15.07 \mu \mathrm{m}$, vs. tfap $2 a ; f o x d 3=71.48 \pm 20.79 \mu \mathrm{m}$ ).

274 comparing wildtype to tfap $2 a$;foxd 3 double mutants; additionally, we observed trajectory timing

275 changes in the absence of neural crest indicating delays in rim involution. Most strikingly,

276 however, was the effect of loss of neural crest on the trajectory and positioning of the nasal RPE.

277 While the other cell populations we tracked reach the correct position and do not show

278 differences in net displacement, the cells which comprise the nasal RPE do not reach the correct

279 position in tfap2a;foxd3 double mutants, as indicated by the change in net displacement.

280 Consistent with neural crest cells migrating in close proximity to the medial side of the optic 
281 vesicle (Fig 1) where cells will either contribute to the RPE or undergo rim involution to

282 contribute to the neural retina, these data demonstrate that the neural crest is critical for

283 regulation of RPE cell movements and rim involution during optic cup morphogenesis.

284

285 Basement membrane formation is disrupted around the RPE in tfap2a;foxd3 double mutants

Having demonstrated a role for neural crest cells in regulating cell movements within the

287 developing optic cup, we sought to determine the underlying molecular mechanism. Although

288 TGF- $\beta$ signaling had been a tantalizing candidate (Fuhrmann et al., 2000; Grocott et al., 2011),

289 our phospho-Smad3 antibody staining results indicated that TGF- $\beta$ signaling to the zebrafish

290 optic cup is not disrupted in the absence of neural crest. Having identified defects in cell

291 movements in many regions of the optic cup, we asked how neural crest might regulate these cell

292 behaviors. Prior work in other systems indicated that mesenchymal cells can regulate epithelial

293 morphogenesis via alterations to the extracellular matrix. We therefore asked whether there

294 might be defects in the ECM surrounding the optic cup when neural crest is lost. By using

295 transmission electron microscopy to directly observe ECM, we visualized assembled basement

296 membranes at the basal surfaces of the brain and RPE, as well as the neural retina and lens in 24

297 hpf control and tfap2a;foxd3 double mutant embryos (Fig 5). Although neural crest cells migrate

298 in the space between the brain and the optic vesicle, the basement membrane lining the

299 developing forebrain in tfap2a;foxd 3 double mutants appears indistinguishable from control

300 embryos (Fig 5A, B); the basement membranes lining the neural retina (Fig 5E, F) and lens (Fig

$3015 \mathrm{G}, \mathrm{H}$ ) also appeared normal in the tfap $2 a$;foxd 3 double mutants compared to wildtype controls.

302 To our surprise, we found that only the basement membrane surrounding the RPE was defective

303 in the tfap2a;foxd3 double mutant (Fig 5D, D'); this basement membrane appears disorganized 
304

305

306

307

308

309

310

311

312

313

314

315

316

317

318

319

320

321

322

323

324

325

326

and discontinuous compared to the same structure in controls (Fig 5C, C'). These results indicate that neural crest cells are required for basement membrane formation around the optic cup, specifically the basement membrane surrounding the RPE. To our knowledge, this is the first indication of neural crest being required to build basement membrane around the developing eye.

The ECM crosslinking protein Nidogen is produced by neural crest and is lost in the tfap2a;foxd3 double mutant

To determine how basement membrane formation is disrupted in the tfap $2 a$;foxd 3 double mutant, we next set out to determine what individual extracellular matrix components might be affected. We asked whether localization or expression of the ECM components laminin-1 and fibronectin were altered in the tfap2a;foxd 3 double mutant. Antibody staining for laminin (Fig S3A-D) and fibronectin (Fig S3E-H) revealed no obvious differences between wildtype and double mutant embryos.

In mouse, the ECM protein nidogen has been found to be necessary for lung and kidney epithelial morphogenesis, yet is provided by surrounding mesenchymal cells (Bader et al., 2005; Ekblom et al., 1994; Kadoya et al., 1997; Willem et al., 2002). Nidogen is an extracellular matrix crosslinking protein which is critical for basement membrane assembly in other systems (Bader et al., 2005; Böse et al., 2006; Ekblom et al., 1994; Kadoya et al., 1997), and, along with laminin-1, is one of two ECM proteins required to be included in the medium to support embryonic-stem-cell-derived optic cup morphogenesis in vitro (Eiraku et al., 2011). We asked whether nidogen localization might be disrupted around the eye in the tfap $2 a$;foxd 3 double mutant. Antibody staining in wildtype embryos revealed that nidogen protein is detected around cells which exhibit mesenchymal morphology and occupy the same space as neural crest cells 
327 migrating between the brain and the optic vesicle at $18 \mathrm{hpf}$ (Fig 6A, A'). Staining is also

328 detectable at the basal surfaces surrounding the RPE, neural retina, and lens at 24 hpf (Fig 6C-

329 C'), consistent with localization within the basement membrane. In contrast, tfap2a;foxd3

330 double mutants that lack neural crest cells also lack nidogen protein between the brain and RPE

331 (Fig 6B, B', D, D'), yet nidogen staining at the basal surfaces of the lens and at the lens-retina

332 interface is unaffected (Fig 6D' '). These data indicate that nidogen is broadly localized to the

333 ECM in wildtype embryos, but is specifically lost around the RPE when neural crest is lost.

In mouse, nidogen expression has been observed in the POM and invaginating lens

Zhu et al., 2017). Despite these observations, the role of nidogen during optic cup morphogenesis

$341 \mathrm{Tg}(\operatorname{sox} 10: G F P)^{b a 4}$ transgenics, we found that both nid1b and nid2a are expressed in sox 10:GFP-

342 positive neural crest cells migrating around the developing optic cup (Fig 7). We also observed

343 both nid1b and nid2a expressed in the overlying ectoderm and the developing lens at 18 and 24

344 hpf, while both are notably absent from the neural retina and RPE. At these same times, nidla is 345 expressed solely in the developing somites (Fig S4A, E, I), while nid2b is detected diffusely

346 throughout the head (Fig S4D, H, L).

348 found in the basal surface of the developing RPE, while the ectoderm and lens placode produce nidogen found surrounding the lens and basal surface of the neural retina. 
Dominant-interfering nidogen disrupts optic cup morphogenesis

mutant was reminiscent of the basement membrane loss observed when nidogen has been

disrupted in mouse models of organogenesis, either through loss of function mutations or 
We previously observed neural crest cells in contact with the optic vesicle as early as

374 12.5 hpf (Fig 1), therefore we performed heat shock from 12-13 hpf to induce WT-Nid1a or DI-

375 Nid1a expression at the onset of neural crest migration around the optic vesicle. We crossed

376

377

378

379

380

381

382

383

384

385

386

387

388

389

390

391

392

393

394

395

zebrafish containing $h s: W T-N i d l a$ or $h s: D I-N i d l a$ transgenes with $\operatorname{Tg}($ bactin2:EGFP-CAAX)z200

transgenics; this enabled us to image the optic cups of $h s:$ WT-Nidla transgene-positive embryos

which had not been heat shocked (Fig 8B, B') as a control. We found that ubiquitous

overexpression of WT-Nid1a slightly, but significantly, impaired invagination $\left(42.3 \pm 1.7^{\circ}\right)$

compared to control embryos $\left(46.8 \pm 1.4^{\circ}\right.$; Fig $\left.8 \mathrm{C}, \mathrm{C}^{\prime}, \mathrm{E}\right)$, and subtly altered lens morphogenesis

such that the resulting lens is not completely spherical. However, overexpression of DI-Nid1a

significantly impaired invagination $\left(32.3 \pm 1.5^{\circ}\right)$, resulting in striking phenotypes including a

severely flattened neural retina and lens (Fig 8 D, D’, E). To determine whether these phenotypes

were due to effects on the optic cup itself or a secondary consequence of disruption to neural

crest migration, we generated double transgenics with sox 10:GFP and either hs:WT-Nidla or

$h s: D I-N i d l a$ to visualize neural crest cells in the presence of WT-Nid1a or DI-Nidla. We find

that ubiquitous, heat shock-induced overexpression of WT-Nidla or DI-Nid1a does not affect

neural crest migration (Fig 8F, G). We conclude, therefore, that DI-Nid1a strongly impairs optic

cup morphogenesis through direct effects on the optic vesicle.

To determine the effects of disrupting nidogen specifically expressed by the neural crest, we attempted to achieve neural crest-specific expression of either WT-Nidla or DI-Nidla by utilizing the Gal4/UAS system. We generated UAS:WT-Nidla and UAS:DI-Nidla transgenic lines and crossed each to $\operatorname{Tg}(\operatorname{sox} 10: \text { Gal4-VP16) })^{\text {el159 }}$ transgenic zebrafish (Das and Crump, 2012).

Unlike ubiquitous, heat-shock induced overexpression, neural crest-specific overexpression of WT-Nid1a did not have an effect on optic cup morphogenesis (data not shown). While neural 
crest-specific expression of DI-Nid1a (sox 10:Gal4-VP16;UAS:DI-Nid1a double transgenic) led

401 Nidla expression (such as when induced from 12-13 hpf via heat shock) does not impair neural

promoter) impairs neural crest migration and/or survival, and thus we were unable to interpret the tissue-specific effects of DI-Nidla expression from the neural crest.

405

406

407

408

\section{Nidogen can partially rescue tfap $2 a$; foxd 3 double mutant optic cup phenotypes}

Nidogen expression in the neural crest, coupled with the phenotypes we observed when we ubiquitously overexpressed DI-Nidla, suggested that disruptions to nidogen's matrix bridging function might be the underlying cause of the morphogenesis defects we observed in mutants where neural crest cells are lost.

We sought to determine whether the optic cup morphogenesis defects we observed in the tfap2a;foxd 3 mutants might indeed be due to a lack of nidogen deposited by neural crest cells. Specifically, we wanted to determine whether expression of WT-Nidla could rescue optic cup morphogenesis in tfap2a;foxd3 double mutants. To test this, we ubiquitously overexpressed WTNid1a in tfap2a;foxd3 double mutants where there would be no neural crest cells present to produce nidogen. We generated tfap2a;foxd3 double mutants that contained both the EGFP-

$417 C A A X$ transgene, as well as the $h s: W T-N i d l a$ transgene. In these embryos, we could visualize the 
419 hpf. In control, non-heat shocked embryos (Fig 9A-B'), we observed retinal invagination defects

420 in tfap2a;foxd3 double mutants compared to their wildtype siblings (Fig 9E), consistent with our

421 previous observations (Fig 2G). To our surprise, wildtype and tfap $2 a$;foxd 3 mutant embryos that

422 were heat shocked from 12-13 hpf looked phenotypically similar. The lenses in either genotype

423 take on a similar, slightly ovoid appearance, and the nasal retina more fully enwraps the lens in

424 tfap2a;foxd3 mutants which overexpress WT-Nid1a, suggesting improved invagination over

425 tfap2a;foxd3 mutant eyes (Fig 9C-D'). When quantified, we indeed observe a significant

426 improvement in the degree of invagination between tfap $2 a ; f$ foxd 3 double mutant eyes $\left(36.8 \pm 1.6^{\circ}\right)$

427 and tfap2a;foxd 3 double mutants which ubiquitiously overexpress WT-Nid1a $\left(39.9 \pm 1.8^{\circ}\right.$; Fig

428 9E). Additionally, we did not detect a difference between heat shocked wildtype and

429 tfap2a;foxd3 embryos when quantifying invagination $\left(39.6 \pm 0.7^{\circ}\right.$ vs $39.9 \pm 1.8^{\circ}$, respectively; Fig

430 9E). From this experiment, we conclude that supplying nidogen can partially bypass the effects

431 of loss of neural crest on optic cup morphogenesis, and that ectopic expression of nidogen from

432 the optic cup and surrounding tissues can partially rescue the invagination defects caused by loss

433 of nidogen producing cells.

\section{Discussion}

$436 \underline{\text { Interactions with neural crest cells regulate optic cup morphogenesis }}$

438 subsequent function. This theme is seen throughout organogenesis, with epithelial-mesenchymal

439 interactions being of particular importance. Developing epithelia frequently require surrounding

440 mesenchyme in order to acquire their mature, functional structures: the lung, kidney, salivary

441 gland, and tooth placode are but a few examples where these interactions are critical (Bader et 
al., 2005; Ekblom et al., 1994; Kadoya et al., 1997; Thesleff, 2003). In the eye, a complex

443 mesenchyme is known to surround the developing optic cup, and mesenchymal cells contribute

444 to later-developing optic tissues such as the hyaloid vasculature and structures within the anterior segment (Gage et al., 2005; James et al., 2016; Soules and Link, 2005; Sowden, 2007). Prior work has established that disruptions to the POM have profound effects on the developing optic

447 cup, such as morphogenetic defects that stretch from the neural retina into the optic stalk and 448 optic fissure (Bassett et al., 2010; Lupo et al., 2011). However, many of these analyses leave 449 many questions unresolved, such as when or where defects arise within the optic cup. The POM 450 is a heterogeneous cell population comprised of both neural crest and mesodermally-derived

451 mesenchymal cells (Gage et al., 2005; Johnston et al., 1979), and specific molecular

452 contributions from either tissue during early optic cup development are poorly understood. A

453 recently published study using elegant ectopic optic vesicle transplants suggested that the POM

454 was dispensable for early optic cup morphogenesis and only required for later stages of optic cup

455 development, specifically the fusion of the optic fissure (Gestri et al., 2018). Here, using the

456 zebrafish tfap2a;foxd3 double mutant which displays a near complete loss of neural crest cells

457 (Arduini et al., 2009; Wang et al., 2011), we demonstrate that the neural crest subpopulation of

458 the POM regulates morphogenesis of the optic cup. Using live imaging, we visualize neural crest

459 cells and find that they migrate around the optic vesicle throughout much of optic cup

460 morphogenesis. Despite relatively mild gross morphological defects, 4-dimensional cell tracking

461 reveals that neural crest cells are required for cell movements within optic vesicle. Specifically,

462 proper RPE cell movements and rim involution depend on the presence of neural crest cells; in

463 the absence of neural crest, cells which contribute to the nasal retina and RPE move significantly

464 farther and faster than in the presence of neural crest. Most notable, however, is the dramatic loss 
465 of basement membrane surrounding the RPE when neural crest is lost, which is likely to underlie

466 these cell movement defects (Figure 10).

The POM has long been observed in close proximity with the developing optic cup, but

469 use to drive optic cup morphogenesis is by modulating the signaling received by the optic vesicle

470 during its development. Zebrafish zic $2 a ; 2 b$ double mutants display disruptions to the periocular

471 neural crest, in addition to molecular and morphological hallmarks of expanded Hedgehog

472 signaling within the optic cup (Sedykh et al., 2017); in tfap2a;foxd3 double mutants however, we

473 do not observe Pax2a (a Hh target gene) expression expanded in a manner consistent with

474 disrupted Hh signaling. In chick, periocular mesenchyme is required for RPE development in a

475 seemingly TGF- $\beta$ dependent fashion (Fuhrmann et al., 2000). Using pSmad3 as a readout, we

476 did not observe a difference in TGF- $\beta$ signaling in the optic cup in tfap $2 a$;foxd 3 double mutants;

477 additionally, neural crest cells are not required for the development of RPE in zebrafish, as we

478 observed pigment granule formation by electron microscopy at $24 \mathrm{hpf}$, and obvious pigmentation

479 of tfap2a;foxd3 mutant eyes at $52 \mathrm{hpf}$. It is possible that that the mesodermal mesenchyme is

480 sufficient for TGF- $\beta$ signaling to the optic cup and RPE induction, or that RPE development

481 could be regulated through different mechanisms in the zebrafish versus the chick. In the future,

482 it will be interesting to dissect the specific roles of the mesodermal mesenchyme in optic cup

483 morphogenesis.

An alternative explanation for the phenotypes we observe in the tfap $2 a$;foxd 3 double 
488 Knight et al., 2005). As such, its loss could affect some aspect of the optic cup morphogenesis

489 through a neural crest-independent effect. However, both tfap $2 a$ and foxd 3 single mutants

490 display optic cup morphogenesis defects that are less severe than those we observe in the

491 tfap2a;foxd3 double mutant. In addition, the lens placode is the sole optic tissue where $\operatorname{tfap} 2 a$ is

492 expressed during optic cup morphogenesis stages, yet the lens is morphologically normal in each

493 of these mutants. Further, we observe disruptions to basement membrane formation along the

494 surface of the RPE which is in direct contact with neural crest cells, but normal basement

495 membrane formation at the basal surface of the retina and lens. These observations suggest that

496 the optic cup morphogenesis defects we observe in the tfap2a;foxd3 double mutants are

497 primarily due to loss of neural crest cells rather than effects on other surrounding tissues.

Neural crest cells promote morphogenesis through ECM assembly

500

Several studies have implicated the ECM surrounding the optic vesicle as a key player in

501 driving optic cup morphogenesis. Fibronectin appears to be critical for formation of the lens

502 (Hayes et al., 2012; Huang et al., 2011), and in retinal organoid culture, the minimal mixture of

503 components needed to elicit optic cup morphogenesis are nodal (a TGF- $\beta$ ligand) and the ECM

504 components laminin-1 and nidogen (Eiraku et al., 2011). Laminin is required for proper cell

505 adhesion of the optic vesicle to the ECM, and disruptions to these adhesions impairs

506 establishment of apicobasal polarity and subsequent morphogenesis (Bryan et al., 2016;

507 Ivanovitch et al., 2013; Nicolás-Pérez et al., 2016; Sidhaye and Norden, 2017). Taken together,

508 these data indicate that the ECM is a critical regulator in formation of the optic cup, and the

509 individual components which comprise the ECM each may regulate specific, non-overlapping

510 aspects of this morphogenetic process. 
512 play a key role in regulating cell movements. Using electron microscopy, we observe a spatially

513 specific, dramatic disruption of basement membrane formation when neural crest is lost. This has

514 allowed us the opportunity to determine the effect of disrupting ECM assembly in a limited

515 domain, without loss of key the basement membrane component laminin. The gross morphology

516 of the optic cup is mildly but reproducibly abnormal, although cell movements in contact with

517 that particular ECM domain are clearly disrupted. We speculate that the intact basement

518 membrane in other parts of the eye is sufficient to support other cell movements underlying optic

519 cup morphogenesis. That being said, comparison of this phenotype with other mutants which

520 disrupt ECM components reveals some notable findings: for example, loss of laminin leads to

521 significant cell death in the prospective RPE domain (Bryan et al., 2016); impairment of

522 basement membrane formation through genetic loss of neural crest cells does not. Further

523 examination of these phenotypes will allow us to identify functions of ECM components that are

524 specifically dependent upon basement membrane assembly. It is also interesting to consider that

525 cells within the developing RPE may rely on the mechanical properties of the surrounding

526 basement membrane in order to migrate and develop correctly. The Hippo signaling pathway is a

527 major cellular mechanotransducer which can function in response to ECM stiffness (Chakraborty

528 et al., 2017), and RPE development is dependent on Yap and Taz, the transcriptional coactivators

529 of the Hippo pathway (Miesfeld et al., 2015). Although RPE development appears normal

530 without assembled basement membrane, it is a tantalizing possibility that Hippo signaling could

531 serve as a molecular link between the basement membrane and optic vesicle cell behaviors.

Although the role of nidogen in other organ systems has been previously investigated, its 
534 components of basement membranes, the mesenchymal cells in the embryonic lung and kidney

535 are those tissues' sole source of nidogens (Ekblom et al., 1994; Kadoya et al., 1997; Senior et al.,

536 1996). Disruptions to nidogen function, either through blocking antibodies or loss-of-function

537 mutations, impedes epithelial morphogenesis of the lung, kidney and developing limb (Bader et

538 al., 2005; Böse et al., 2006; Ekblom et al., 1994; Kadoya et al., 1997). Here, we demonstrate that

539 neural crest cells produce the nidogen which is deposited along the basal surface of the RPE, and

540 that neural crest cells are required for proper assembly of the basement membrane along this

541 surface. Impairing nidogen function, either through loss of neural crest cells or through

542 expression of a dominant-interfering form of Nidla, leads to defects in optic cup morphogenesis.

543 Intriguingly, ubiquitous overexpression of full-length Nidla also causes slight defects in optic

544 cup invagination, suggesting that an optimal level of nidogen is required for proper ECM

545 function. Consistent with this, loss of the nidogen-binding site in the laminin $\gamma 1$ chain results in

546 higher penetrance of renal defects than when both nidogen 1 and 2 are lost (Bader et al., 2005;

547 Willem et al., 2002); this was predicted to be due to an increase of free nidogen incapable of

548 binding to laminin, which could sequester other components of the ECM and interfere with other

549 matrix interactions (Bader et al., 2005).

550 How the addition of nidogen to the ECM surrounding the optic cup promotes the

551 morphogenetic movements required for optic cup morphogenesis is unclear. While both laminin

552 and fibronectin were still detectable around the RPE in the absence of neural crest cells, we did

553 not detect a normal basement membrane at this site. This finding is consistent with mouse

554 nidogen mutants which show similar basement membrane defects around the developing kidney,

555 lung, and heart, despite retention of other ECM components (Bader et al., 2005). Proper

556 attachment to the ECM is required for many aspects of optic cup morphogenesis including RPE 
557 cell movements and rim involution (Bryan et al., 2016; Hayes et al., 2012; Martinez-Morales et

558 al., 2009; Sidhaye and Norden, 2017), two processes which we show to be disrupted in the

559 absence of neural crest. We propose that through deposition of nidogen into the ECM, neural

560 crest cells generate a restrictive environment which enables the correct movements of RPE cells

561 and migration of the cells which move around the rim of the optic vesicle and into the

562 developing neural retina; the basement membrane surrounding the RPE serves as a handbrake

563 which slows optic vesicle cell movements and ensures their migration to the correct place at the

564 correct time during optic cup morphogenesis. Disruptions to nidogen function, either through

565 loss of neural crest cells, expression of a dominant-interfering form of nidogen, or

566 overexpression of wildtype nidogen, lead to defects in optic cup morphogenesis. It will be

567 interesting to investigate whether cells in the developing optic cup can still properly adhere to the

568 remaining ECM components in the absence of neural crest cells. Further, growth factors and

569 signaling molecules such as FGFs and BMPs are regulated through deposition into the ECM, and

570 correct assembly of the basement membrane may be required to properly regulate these signaling

571 pathways during optic cup morphogenesis; these will be interesting signaling pathways to

572 investigate in the context of disrupted basement membrane assembly. 


\section{Materials and methods}

574 Zebrafish lines

575 Embryos from the following mutant and transgenic lines were raised at $28.5^{\circ} \mathrm{C}$ and

576 staged according to hours post fertilization and morphology (Kimmel et al., 1995).

577 Mutant alleles:

578 tfap $2 a^{t s 213}$;foxd $3^{z d f 10}$ (Arduini et al., 2009). The alyron ${ }^{224}$ allele contains a $\mathrm{C}$ to A transversion in

579 the coding sequence of the pafl gene, resulting in a premature stop mutation at tyrosine 281

580 (Y281*) (Mick Jurynec and David Grunwald, personal communication).

581 Transgenic alleles:

$582 \operatorname{Tg}(\operatorname{sox} 10: m e m R F P)^{v u 234}$ (Kirby et al., 2006), $\operatorname{Tg}(\operatorname{sox} 10: G F P)^{b a 4}$ (Dutton et al., 2008),

583 Tg(bactin2:EGFP-CAAX) ${ }^{z 200}, \operatorname{Tg}(\text { hsp 70:lyn-mCherry-2A-WT-Nid1a })^{z 202}, \operatorname{Tg}($ hsp $70: l y n-$

584 mCherry-2A-DI-Nidla) ${ }^{z 203}$.

585

586

Construction of Nidla transgenic lines

Tg(hsp70:lyn-mCherry-2A-WT-Nidla), a.k.a. hs:WT-Nidla and Tg(hsp70:lyn-mCherry-

589 recombination. IMAGE Clone ID 8000296 (GE Dharmacon, Chicago, IL) was used as the

590 template to PCR amplify cDNAs encoding wildtype and dominant-interfering Nidla, these were

591 ligated into pCS2FA prior to Gateway cloning. PCR primers were used to introduce the PTV-2A 592 peptide (Provost et al., 2007) on the 5' end, and the SV40 late poly-adenylation signal on the 3'

593 end of the zebrafish nidla cDNA. Gateway 3' entry clones were generated via BP recombination

594 and subsequently LR recombined into the pDEST-Tol2-CG2 destination vector which contains

595 an myl7:EGFP expression cassette as a transgenesis marker (Kwan et al., 2007). 25 pg plasmid 
596 DNA was microinjected along with 50 pg mRNA encoding Tol2 transposase into single-cell

597 wildtype embryos and screened for myl7:EGFP expression. Fluorescent embryos were raised to

598 adulthood and outcrossed to generate stable transgenic lines.

599

600 Heat shocks

601 Embryos were transferred from a $28.5^{\circ} \mathrm{C}$ incubator and immediately overlaid with fresh,

602 preheated $39^{\circ} \mathrm{C} \mathrm{E} 3$. Embryos were incubated at $39^{\circ} \mathrm{C}$ for one hour on an Echotherm heating plate

603 (Torrey Pines Scientific, Carlsbad, CA). Embryos were then transferred back to a $28.5^{\circ} \mathrm{C}$

604 incubator and grown to the indicated stage.

605

606 Allele identification/genotyping

607 All mutant alleles were PCR genotyped using either CAPS or dCAPS techniques (Neff et al.,

608 1998). tfap $2 a^{t s 213}$ : Forward (5'-CGCTCAGGTCTTATAAATAGGCTACTAATAATGTTAC-

609 3'), Reverse (5'- CTGAGAGGTGGCTATTTCCCGTTAAGATTCG-3'), mutant allele is cut

610 with BlpI.

611 foxd $3^{z d f 10}:$ dCAPS Forward (5'-

612 CGACTGCTTCGTCAAGATCCCACGGGAACCGGGCAACCCGGGCAAAGGCAACTACT

613 GGACCCTCGACCCCCAGTCGGAAAATAT-3'), Reverse (5’-

614 CAGGGGGAATGTACGGGTACTGC-3'), mutant allele is cut with SspI.

615 paf1 ${ }^{24}$ : Forward (5'-GTTCAGAGGTATGATGGATGAGG-3'), Reverse (5'-

616 GTATGCAGCTTTATGAAAACACTC-3'), wildtype band is cut with NspI. 
RNA synthesis and injections

618 Capped mRNAs were synthesized using linearized pCS2 templates (pCS2-EGFP-CAAX,

619 pCS2FA-H2A.F/Z-mCherry), the mMessage mMachine SP6 kit (AM1340, Invitrogen), purified

620 using the Qiagen RNeasy Mini Kit (Qiagen, Hilden, Germany) and ethanol precipitated. 150-250

621 pg of each mRNA were microinjected into the cell of one-cell stage embryos. EGFP-CAAX

622 mRNA was injected to visualize cell membranes, H2A.F/Z-mCherry mRNA was injected to

623 visualize nuclei.

624

625

Antibody staining

626

Embryos were fixed at the indicated stage in 4\% paraformaldehyde, permeabilized in

627

PBST (PBS $+0.5 \%$ Triton X-100), and blocked in PBST $+2 \%$ bovine serum albumin. Antibodies

628 and concentrations are as follows: anti-Pax2a (GTX128127, Genetex, Irvine, CA), 1:200; anti-

629 pSmad3 (ab52903, Abcam, Cambridge, MA), 1:200; anti-Nidogen/Entactin (ab14511, Abcam),

630 1:100; anti-Laminin 1 (L9393, Sigma, St. Louis, MO), 1:100; anti-Fibronectin (F3648, Sigma),

631 1:100; anti-GFP (A10262, Invitrogen), 1:200. Secondary antibodies used were Alexa Fluor 488

632 goat anti-mouse (A-11001, Invitrogen), Alexa Fluor 488 goat anti-rabbit (A-11008, Invitrogen),

633 Alexa Fluor 488 goat anti-chicken (A-11039, Invitrogen) and incubated at 1:200. Nuclei were

634 detected by incubation with $1 \mu \mathrm{M}$ TOPRO-3 iodide (T3605, Invitrogen). Embryos were cleared

635 through a series of 30\%/50\%/70\% glycerol (in PBS) prior to imaging.

636

637 In situ hybridization

638 Embryos were fixed at the indicated stage in $4 \%$ paraformaldehyde overnight at $4{ }^{\circ} \mathrm{C}$ and

639 dehydrated in 100\% methanol. Color in situ hybridizations were performed similar to (Thisse 
and Thisse, 2008). Fluorescent in situ hybridizations were carried out as described previously

641 (Lauter et al., 2014; Leerberg et al., 2017). Anti-GFP labeling and detection was performed after

642 in situ hybridization and tyramide signal amplification.

643

In situ probes were synthesized from linearized pBluescript II SK+ templates (pBSII-

644 Nid1a, pBSII-Nid1b, pBSII-Nid2a, pBSII-Nid2b) using T3 or T7 polymerases and DIG labeling

mix (11277073910, Roche, Basel, Switzerland). All four probe sequences were synthesized (IDT

646 gBlocks, IDT, San Jose, CA) and ligated into pBluescript II SK+.

647

648

Light Microscopy

649

For timelapse imaging, $12 \mathrm{hpf}$ embryos were embedded in 1.6\% low-melt agarose (in E3)

650

in DeltaT dishes (Bioptechs, \#0420041500 C), E3 was overlaid and the dish covered to prevent

651 evaporation. For antibody stained or fluorescent in situ hybridization imaging, embryos were

652 embedded in 1\% low-melt agarose (in PBS) in Pelco glass-bottom dishes (\#14027, Ted Pella,

653 Redding, CA), PBS was overlaid to prevent evaporation.

654 Confocal images were acquired using a Zeiss LSM710 laser scanning confocal

655 microscope. For timelapse imaging, datasets were acquired using the following parameters: $63 \mathrm{z}-$

656 sections, $2.10 \mu \mathrm{m}$ z-step, 40x water-immersion objective (1.2 NA). Time between z-stacks was 3

657 minutes 30 seconds (Movies S1, S2), 2 minutes 45 seconds (Movies S4, S6, S8), and 2 minutes

65830 seconds (Movies S5, S7, S9). For all timelapse and antibody imaging, datasets were acquired

659 without knowledge of embryo genotype. Embryos were de-embedded and genotyped after

660 imaging was completed.

661 Brightfield images were acquired using an Olympus SZX16 stereomicroscope with either 662 an Olympus DP26 or UC90 camera. 
663

664

665

666

667

668

669

670

671

672

673

674

675

676

677

678

679

680

681

682

683

684 685 nuclei were visualized using H2A.F/Z-mCherry.

\section{Transmission Electron Microscopy} prior to fixation and used for genotyping.

\section{Image processing and analysis}

24 hpf embryos were fixed, stained and embedded using the microwave-assisted tissue processing protocol described in (Czopka and Lyons, 2011). Tails were dissected from embryos

Our tissue sampling and analytical techniques have been described previously in detail (Anderson et al., 2011a, 2011b; Lauritzen et al., 2013; Marc et al., 2013, 2014).

The tissues were osmicated for $60 \mathrm{~min}$ in $0.5 \% \mathrm{OsO} 4$ in $0.1 \mathrm{M}$ cacodylate buffer, processed in maleate buffer for en bloc staining with uranyl acetate, and processed for resin embedding. The epoxy resin bloc with zebrafish tissue was sectioned in the horizontal plane at 70-90 nm onto polyvinyl formal resin coated copper slot grids for transmission electron microscopy (TEM) (Lauritzen et al., 2013; Marc and Jones, 2002).

Each TEM section was imaged on a JEOL JEM-1400 transmission electron microscope at 20,000x and stored in 16- and 8-bit versions, as well as image pyramids of optimized tiles for web visualization with the Viking viewer (Anderson et al., 2011a, 2011b). Each image was captured as an array of image tiles at roughly 500-800 tiles/slice with $15 \%$ overlap.

Images were processed using ImageJ. Volume rendering was performed using FluoRender (Wan et al., 2009, 2017). For lateral view 3D rendering of the optic cup, the ectoderm was digitally erased in ImageJ prior to visualization in FluoRender. Invagination angles were measured as previously described (Bryan et al., 2016) and shown in Fig 2G.

Individual cell tracking was performed as described in (Kwan et al., 2012) using LongTracker; 


\section{Acknowledgements}

688 We are grateful to Rodney Stewart, David Grunwald, and Mick Jurynec for reagents, and the

689 University of Utah Centralized Zebrafish Animal Resource for zebrafish husbandry. Thanks to

690 members of the Kwan lab for useful discussions and critical reading of the manuscript. This

691 work was supported by grants from the NEI/NIH to K. M. K. (R01 EY025378, R01 EY025780),

692 to B. W. J. (R01 EY015128), and to the Moran Eye Center Vision Core (P30 EY014800). C. D.

693 B. was supported by the University of Utah Developmental Biology Training Grant (NIH

694 T32HD007491). 


\section{References}

696

697

698

699

700

701

702

703

704

705

706

707

708

709

710

711

712

713

714

715

716

717

718

719

720

721

722

723

724

725

726

727

728

729

730

731

732

733

734

735

736

737

738

739
Akanuma, T., Koshida, S., Kawamura, A., Kishimoto, Y., and Takada, S. (2007). Pafl complex homologues are required for Notch-regulated transcription during somite segmentation. EMBO Rep. 8, 858-863.

Anderson, J.R., Jones, B.W., Watt, C.B., Shaw, M. V, Yang, J.H., Demill, D., Lauritzen, J.S., Lin, Y., Rapp, K.D., Mastronarde, D., et al. (2011a). Exploring the retinal connectome. Mol Vis $17,355-379$.

Anderson, J.R., Mohammed, S., Grimm, B., Jones, B.W., Koshevoy, P., Tasdizen, T., Whitaker, R., and Marc, R.E. (2011b). The Viking viewer for connectomics: Scalable multi-user annotation and summarization of large volume data sets. J. Microsc. 241, 13-28.

Arduini, B.L., Bosse, K.M., and Henion, P.D. (2009). Genetic ablation of neural crest cell diversification. Development 136, 1987-1994.

Bader, B.L., Smyth, N., Nedbal, S., Baranowsky, A., Mokkapati, S., Miosge, N., Murshed, M., Nischt, R., Baranowsky, A., Mokkapati, S., et al. (2005). Compound Genetic Ablation of Nidogen 1 and 2 Causes Basement Membrane Defects and Perinatal Lethality in Mice. Mol. Cell. Biol. 25, 6846-6856.

Bassett, E.A., Williams, T., Zacharias, A.L., Gage, P.J., Fuhrmann, S., and West-Mays, J.A. (2010). AP-2a knockout mice exhibit optic cup patterning defects and failure of optic stalk morphogenesis. Hum. Mol. Genet. 19, 1791-1804.

Bohnsack, B.L., Kasprick, D.S., Kish, P.E., Goldman, D., and Kahana, A. (2012). A zebrafish model of Axenfeld-Rieger syndrome reveals that pitx 2 regulation by Retinoic Acid is essential for ocular and craniofacial development. Investig. Ophthalmol. Vis. Sci. 53, 7-22.

Böse, K., Nischt, R., Page, A., Bader, B.L., Paulsson, M., and Smyth, N. (2006). Loss of nidogen-1 and -2 results in syndactyly and changes in limb development. J. Biol. Chem. 281, 39620-39629.

Bryan, C.D., Chien, C. Bin, and Kwan, K.M. (2016). Loss of laminin alpha 1 results in multiple structural defects and divergent effects on adhesion during vertebrate optic cup morphogenesis. Dev. Biol. 416, 324-337.

Chakraborty, S., Njah, K., Pobbati, A. V., Lim, Y.B., Raju, A., Lakshmanan, M., Tergaonkar, V., Lim, C.T., and Hong, W. (2017). Agrin as a Mechanotransduction Signal Regulating YAP through the Hippo Pathway. Cell Rep. 18, 2464-2479.

Cretekos, C.J., and Grunwald, D.J. (1999). Alyron, an Insertional Mutation Affecting Early Neural Crest Development in Zebrafish. Dev. Biol. 210, 322-338.

Czopka, T., and Lyons, D.A. (2011). Dissecting Mechanisms of Myelinated Axon Formation Using Zebrafish. In The Zebrafish: Disease Models and Chemical Screens, (Elsevier Inc.), pp. 25-62.

Das, A., and Crump, J.G. (2012). Bmps and Id2a act upstream of twist 1 to restrict ectomesenchyme potential of the cranial neural crest. PLoS Genet. 8.

Dong, L.J., and Chung, A.E. (1991). The Expression of the Genes for Entactin, Laminin-a, Laminin-B1 and Laminin-B2 in Murine Lens Morphogenesis and Eye Development.

Differentiation 48, 157-172.

Dutton, J.R., Antonellis, A., Carney, T.J., Rodrigues, F.S., Pavan, W.J., Ward, A., and Kelsh, R.N. (2008). An evolutionarily conserved intronic region controls the spatiotemporal expression of the transcription factor Sox10. BMC Dev. Biol. 8, 105.

Eberhart, J.K., He, X., Swartz, M.E., Yan, Y.L., Song, H., Boling, T.C., Kunerth, A.K., Walker, 
M.B., Kimmel, C.B., and Postlethwait, J.H. (2008). MicroRNA Mirn140 modulates Pdgf signaling during palatogenesis. Nat. Genet. 40, 290-298. Eiraku, M., Takata, N., Ishibashi, H., Kawada, M., Sakakura, E., Okuda, S., Sekiguchi, K., Adachi, T., and Sasai, Y. (2011). Self-organizing optic-cup morphogenesis in three-dimensional culture. Nature 472, 51-56.

Ekblom, P., Ekblom, M., Fecker, L., Klein, G., Zhang, H.Y., Kadoya, Y., Chu, M.L., Mayer, U., and Timpl, R. (1994). Role of mesenchymal nidogen for epithelial morphogenesis in vitro. Development 120, 2003-2014.

Fox, J.W., Mayer, U., Nischt, R., Aumailley, M., Reinhardt, D., Wiedemann, H., Mann, K., Timpl, R., Krieg, T., and Engel, J. (1991). Recombinant nidogen consists of three globular domains and mediates binding of laminin to collagen type IV. EMBO J. 10, 3137-3146. Fuhrmann, S., Levine, E.M., and Reh, T.A. (2000). Extraocular mesenchyme patterns the optic vesicle during early eye development in the embryonic chick. Development 127, 4599-4609. Gage, P.J., Rhoades, W., Prucka, S.K., and Hjalt, T. (2005). Fate maps of neural crest and mesoderm in the mammalian eye. Investig. Ophthalmol. Vis. Sci. 46, 4200-4208.

Gestri, G., Osborne, R.J., Wyatt, A.W., Gerrelli, D., Gribble, S., Stewart, H., Fryer, A., Bunyan, D.J., Prescott, K., Collin, J.R.O., et al. (2009). Reduced TFAP2A function causes variable optic fissure closure and retinal defects and sensitizes eye development to mutations in other morphogenetic regulators. Hum. Genet. 126, 791-803.

Gestri, G., Bazin-Lopez, N., Scholes, C., and Wilson, S.W. (2018). Cell Behaviors during Closure of the Choroid Fissure in the Developing Eye. Front. Cell. Neurosci. 12, 1-12. Grocott, T., Johnson, S., Bailey, A.P., and Streit, A. (2011). Neural crest cells organize the eye via TGF- $\beta$ and canonical Wnt signalling. Nat. Commun. 2, 266-269. Hayes, J.M., Hartsock, A., Clark, B.S., Napier, H.R.L., Link, B.A., and Gross, J.M. (2012). Integrin 5/Fibronectin 1 and focal adhesion kinase are required for lens fiber morphogenesis in zebrafish. Mol. Biol. Cell 23, 4725-4738. Heermann, S., Schütz, L., Lemke, S., Krieglstein, K., and Wittbrodt, J. (2015). Eye morphogenesis driven by epithelial flow into the optic cup facilitated by modulation of bone morphogenetic protein. Elife 4, 1-17.

Hendrix, R.W., and Zwaan, J. (1975). The matrix of the optic vesicle-presumptive lens interface during induction of the lens in the chicken embryo. J. Embryol. Exp. Morphol. 33, 1023-1049. Hero, I. (1990). Optic fissure closure in the normal cinnamon mouse: An ultrastructural study. Investig. Ophthalmol. Vis. Sci. 31, 197-216.

Hero, I., Farjah, M., and Scholtz, C.L. (1991). The prenatal development of the optic fissure in colobomatous microphthalmia. Investig. Ophthalmol. Vis. Sci. 32, 2622-2635.

Hilfer, S.R. (1983). Development of the eye of the chick embryo. Scan. Electron Microsc. 13531369.

Huang, J., Rajagopal, R., Liu, Y., Dattilo, L.K., Shaham, O., Ashery-Padan, R., and Beebe, D.C. (2011). The mechanism of lens placode formation: A case of matrix-mediated morphogenesis. Dev. Biol. 355, 32-42.

Ivanovitch, K., Cavodeassi, F., and Wilson, S.W. (2013). Precocious acquisition of neuroepithelial character in the eye field underlies the onset of eye morphogenesis. Dev. Cell 27, 293-305.

James, A., Lee, C., Williams, A.M., Angileri, K., Lathrop, K.L., and Gross, J.M. (2016). The hyaloid vasculature facilitates basement membrane breakdown during choroid fissure closure in the zebrafish eye. Dev. Biol. 419, 262-272. 
Johnston, M.C., Noden, D.M., Hazelton, R.D., Coulombre, J.L., and Coulombre, A.J. (1979). Kadoya, Y., Salmivirta, K., Talts, J.F., Kadoya, K., Mayer, U., Timpl, R., and Ekblom, P. (1997). Importance of nidogen binding to laminin $\gamma 1$ for branching epithelial morphogenesis of the submandibular gland. Development 124, 683-691.

Kheradmand, F., Rishi, K., and Werb, Z. (2002). Signaling through the EGF receptor controls lung morphogenesis in part by regulating MT1-MMP-mediated activation of gelatinase A/MMP2. J. Cell Sci. 115, 839-848. embryonic development of the zebrafish. Dev. Dyn. 203, 253-310.

Kirby, B.B., Takada, N., Latimer, A.J., Shin, J., Carney, T.J., Kelsh, R.N., and Appel, B. (2006). In vivo time-lapse imaging shows dynamic oligodendrocyte progenitor behavior during zebrafish development. Nat. Neurosci. 9, 1506-1511. signals from the ectoderm regulate craniofacial development in the zebrafish embryo.

801 Development 132, 3127-3138.

802 Kudoh, T., Tsang, M., Hukriede, N.A., Chen, X., Dedekian, M., Clarke, C.J., Kiang, A., Schultz, 803 S., Epstein, J.A., Toyama, R., et al. (2001). A gene expression screen in zebrafish embryogenesis. Genome Res. 11, 1979-1987.

Kwan, K.M. (2014). Coming into focus: The role of extracellular matrix in vertebrate optic cup morphogenesis. Dev. Dyn. 243, 1242-1248.

807 Kwan, K.M., Fujimoto, E., Grabher, C., Mangum, B.D., Hardy, M.E., Campbell, D.S., Parant, 808 J.M., Yost, H.J., Kanki, J.P., and Chien, C. Bin (2007). The Tol2kit: A multisite gateway-based construction Kit for Tol2 transposon transgenesis constructs. Dev. Dyn. 236, 3088-3099. Kwan, K.M., Otsuna, H., Kidokoro, H., Carney, K.R., Saijoh, Y., and Chien, C.-B. (2012). A complex choreography of cell movements shapes the vertebrate eye. Development 139, 359-372. Langenbacher, A.D., Nguyen, C.T., Cavanaugh, A.M., Huang, J., Lu, F., and Chen, J.N. (2011). The PAF1 complex differentially regulates cardiomyocyte specification. Dev. Biol. 353, 19-28. Langenberg, T., Kahana, A., Wszalek, J.A., and Halloran, M.C. (2008). The eye organizes neural crest cell migration. Dev. Dyn. 237, 1645-1652.

Lauritzen, J.S., Anderson, J.R., Jones, B.W., Watt, C.B., Mohammed, S., Hoang, J. V., and Marc, R.E. (2013). ON Cone Bipolar Cell Axonal Synapses in the OFF Inner Plexiform Layer of the Rabbit Retina. J. Comp. Neurol. 521, 977-1000.

Lauter, G., Söll, I., and Hauptmann, G. (2014). Sensitive Whole-Mount Fluorescent In Situ Hybridization in Zebrafish Using Enhanced Tyramide Signal Amplification. Methods Mol. Biol. 1082, 175-185.

Lee, J., Willer, J.R., Willer, G.B., Smith, K., Gregg, R.G., and Gross, J.M. (2008). Zebrafish blowout provides genetic evidence for Patched1-mediated negative regulation of Hedgehog signaling within the proximal optic vesicle of the vertebrate eye. Dev. Biol. 319, 10-22.

Leerberg, D.M., Sano, K., and Draper, B.W. (2017). Fibroblast growth factor signaling is required for early somatic gonad development in zebrafish. PLOS Genet. 1-28.

Li, W., and Cornell, R.A. (2007). Redundant activities of Tfap2a and Tfap2c are required for neural crest induction and development of other non-neural ectoderm derivatives in zebrafish embryos. Dev. Biol. 304, 338-354. 
Lupo, G., Gestri, G., O’Brien, M., Denton, R.M., Chandraratna, R.A.S., Ley, S. V, Harris, W.A., and Wilson, S.W. (2011). Retinoic acid receptor signaling regulates choroid fissure closure through independent mechanisms in the ventral optic cup and periocular mesenchyme. Proc. Natl. Acad. Sci. U. S. A. 108, 8698-8703. Marc, R.E., and Jones, B.W. (2002). Molecular phenotyping of retinal ganglion cells. J. Neurosci. 22, 413-427. Marc, R.E., Jones, B.W., Watt, C.B., Anderson, J.R., Sigulinsky, C., and Lauritzen, S. (2013). Retinal connectomics: Towards complete, accurate networks. Prog. Retin. Eye Res. 37, 141-162. Marc, R.E., Anderson, J.R., Jones, B.W., Sigulinsky, C.L., and Lauritzen, J.S. (2014). The AII amacrine cell connectome: a dense network hub. Front. Neural Circuits 8, 1-13. Martinez-Morales, J.R., Rembold, M., Greger, K., Simpson, J.C., Brown, K.E., Quiring, R., Pepperkok, R., Martin-Bermudo, M.D., Himmelbauer, H., and Wittbrodt, J. (2009). Ojoplanomediated basal constriction is essential for optic cup morphogenesis. Development 136, $2165-$ 2175.

Miesfeld, J.B., Gestri, G., Clark, B.S., Flinn, M.A., Poole, R.J., Bader, J.R., Besharse, J.C., Wilson, S.W., and Link, B.A. (2015). Yap and Taz regulate retinal pigment epithelial cell fate. Development 142, 3021-3032.

Neff, M.M., Neff, J.D., Chory, J., and Pepper, A.E. (1998). dCAPS, a simple technique for the genetic analysis of single nucleotide polymorphisms: experimental applications in Arabidopsis thaliana genetics. Plant J. 14, 387-392.

Nelson, D.A., and Larsen, M. (2015). Heterotypic control of basement membrane dynamics during branching morphogenesis. Dev. Biol. 401, 103-109.

Nguyen, C.T., Langenbacher, A., Hsieh, M., and Chen, J.N.O. (2010). The PAF1 complex component Leol is essential for cardiac and neural crest development in zebrafish. Dev. Biol. 341, 167-175.

Nicolás-Pérez, M., Kuchling, F., Letelier, J., Polvillo, R., Wittbrodt, J., and Martínez-Morales, J.R. (2016). Analysis of cellular behavior and cytoskeletal dynamics reveal a constriction mechanism driving optic cup morphogenesis. Elife 5, 1-24.

Peterson, P.E., Pow, C.S., Wilson, D.B., and Hendrickx, A.G. (1995). Localisation of glycoproteins and glycosaminoglycans during early eye development in the macaque. J. Anat. 186 ( Pt 1, 31-42.

Picker, A., Cavodeassi, F., Machate, A., Bernauer, S., Hans, S., Abe, G., Kawakami, K., Wilson, S.W., and Brand, M. (2009). Dynamic coupling of pattern formation and morphogenesis in the developing vertebrate retina. PLoS Biol. 7. Provost, E., Rhee, J., and Leach, S.D. (2007). Viral 2A peptides allow expression of multiple proteins from a single ORF in transgenic zebrafish embryos. Genesis 45, 625-629.

Pujuguet, P., Simian, M., Liaw, J., Timpl, R., Werb, Z., and Bissell, M.J. (2000). Nidogen-1 regulates laminin-1-dependent mammary-specific gene expression. J. Cell Sci. 113, 849-858. Reinhardt, D., Mann, K., Nischt, R., Fox, J.W., Chu, M.L., Krieg, T., and Timpl, R. (1993). Mapping of nidogen binding sites for collagen type IV, heparan sulfate proteoglycan, and zinc. J. Biol. Chem. 268, 10881-10887.

Schmitt, E.A., and Dowling, J.E. (1994). Early eye morphogenesis in the zebrafish, Brachydanio rerio. J. Comp. Neurol. 344, 532-542.

Schook, P. (1980). Morphogenetic movements during the early development of the chick eye. A light microscopic and spatial reconstructive study. Acta Morphol. Neerl. Scand. 18, 1-30. Sedykh, I., Yoon, B., Roberson, L., Moskvin, O., Dewey, C.N., and Grinblat, Y. (2017). 
Zebrafish zic2 controls formation of periocular neural crest and choroid fissure morphogenesis. Dev. Biol. 429, 92-104.

Senior, R.M., Griffin, G.L., Mudd, M.S., Moxley, M.A., Longmore, W.J., and Pierce, R.A. (1996). Entactin expression by rat lung and rat alveolar epithelial cells. Am. J. Respir. Cell Mol. Biol. 14, 239-247.

Sidhaye, J., and Norden, C. (2017). Concerted action of neuroepithelial basal shrinkage and active epithelial migration ensures efficient optic cup morphogenesis. Elife 6, 1-29.

Soules, K.A., and Link, B.A. (2005). Morphogenesis of the anterior segment in the zebrafish eye. BMC Dev. Biol. 5, 12.

Sowden, J.C. (2007). Molecular and developmental mechanisms of anterior segment dysgenesis. Eye (Lond). 21, 1310-1318.

Svoboda, K.K., and O'Shea, K.S. (1987). An analysis of cell shape and the neuroepithelial basal lamina during optic vesicle formation in the mouse embryo. Development 100, 185-200. Thesleff, I. (2003). Epithelial-mesenchymal signalling regulating tooth morphogenesis. J. Cell Sci. 116, 1647-1648.

Thisse, B., and Thisse, C. (2004). Fast Release Clones: A High Throughput Expression Analysis. ZFIN Direct Data Submiss.

Thisse, C., and Thisse, B. (2008). High-resolution in situ hybridization to whole-mount zebrafish embryos. Nat. Protoc. 3, 59-69.

Tuckett, F., and Morriss-Kay, G.M. (1986). The distribution of fibronectin, laminin and entactin in the neurulating rat embryo studied by indirect immunofluorescence. J. Embryol. Exp. Morphol. 94, 95-112.

Walls, G.L. (1942). The vertebrate eye and its adaptive radiation (Hafner Publishing Company). Wan, Y., Otsuna, H., Chien, C. Bin, and Hansen, C. (2009). An interactive visualization tool for multi-channel confocal microscopy data in neurobiology research. IEEE Trans. Vis. Comput. Graph. 15, 1489-1496. Wan, Y., Otsuna, H., Holman, H.A., Bagley, B., Ito, M., Lewis, A.K., Colasanto, M., Kardon, G., Ito, K., and Hansen, C. (2017). FluoRender: Joint freehand segmentation and visualization for many-channel fluorescence data analysis. BMC Bioinformatics 18, 1-15. Wang, W.-D., Melville, D.B., Montero-Balaguer, M., Hatzopoulos, A.K., and Knapik, E.W. (2011). Tfap2a and Foxd3 regulate early steps in the development of the neural crest progenitor population. Dev. Biol. 360, 173-185.

Weiss, O., Kaufman, R., Michaeli, N., and Inbal, A. (2012). Abnormal vasculature interferes with optic fissure closure in lmo2 mutant zebrafish embryos. Dev. Biol. 369, 191-198. Wells, K.L., Gaete, M., Matalova, E., Deutsch, D., Rice, D., and Tucker, A.S. (2013). Dynamic relationship of the epithelium and mesenchyme during salivary gland initiation: the role of Fgf10. Biol. Open 2, 981-989.

Willem, M., Miosge, N., Halfter, W., Smyth, N., Jannetti, I., Burghart, E., Timpl, R., and Mayer, U. (2002). Specific ablation of the nidogen-binding site in the laminin $\gamma 1$ chain interferes with kidney and lung development. Development 129, 2711-2722.

Williams, A.L., and Bohnsack, B.L. (2015). Neural crest derivatives in ocular development: Discerning the eye of the storm. Birth Defects Res. Part C - Embryo Today Rev. 105, 87-95. Zhu, P., Ma, Z., Guo, L., Zhang, W., Zhang, Q., Zhao, T., Jiang, K., Peng, J., and Chen, J. (2017). Short body length phenotype is compensated by the upregulation of nidogen family members in a deleterious nidla mutation of zebrafish. J. Genet. Genomics 44, 553-556. 


\section{Figures}

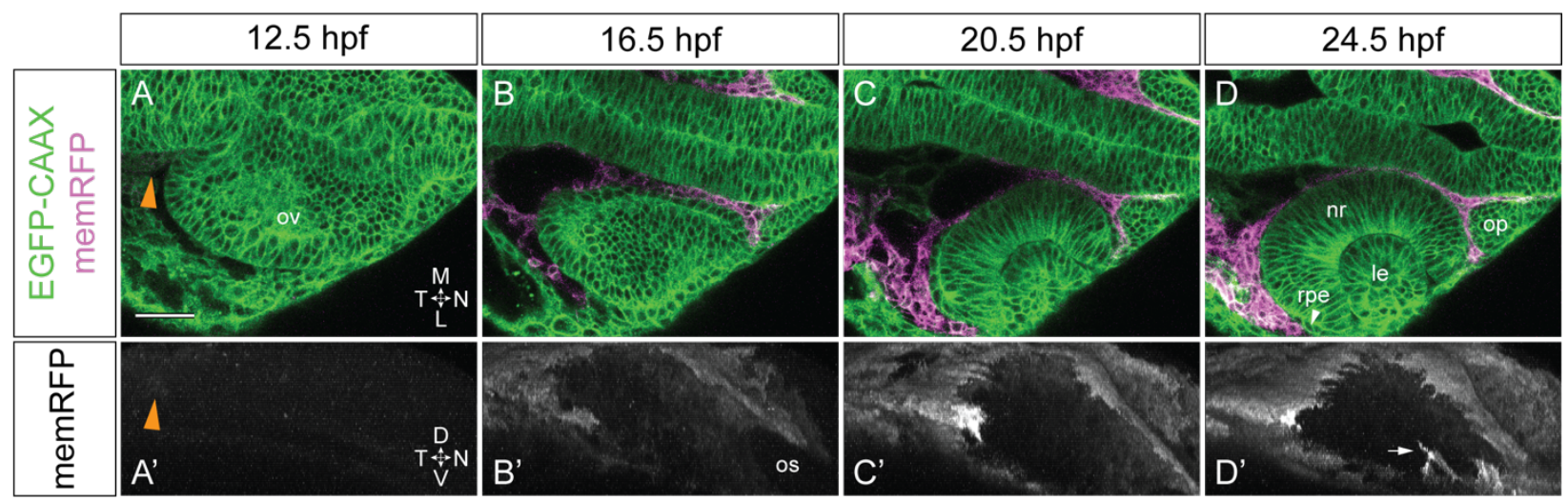

Figure 1. Neural crest is in contact with the optic vesicle throughout optic cup morphogenesis. Live imaging time series from 12.5-24.5 hpf of a $\operatorname{Tg}($ bactin2:EGFP-CAAX); $\operatorname{Tg}(\operatorname{sox} 10: m e m R F P)$ double transgenic embryo. (A-D) Dorsal view, single confocal sections from a 4D dataset. (A'D') Lateral view, 3D rendering of the RFP channel from the same dataset as shown in (A-D). Orange arrowheads in A, A' indicate neural crest cells beginning to express memRFP. White arrowhead in D indicates retinal pigment epithelium between neural crest and neural retina. White arrow in D' indicates neural crest-derived cells entering the optic fissure. Scale bar, 50 $\mu \mathrm{m} . \Delta \mathrm{T}$ between z-stacks, 3.5 minutes. ov, optic vesicle; os, optic stalk; nr, neural retina; rpe, retinal pigment epithelium; le, lens; op, olfactory placode. M, medial; L, lateral; D, dorsal; V, ventral; N, nasal; T, temporal. 


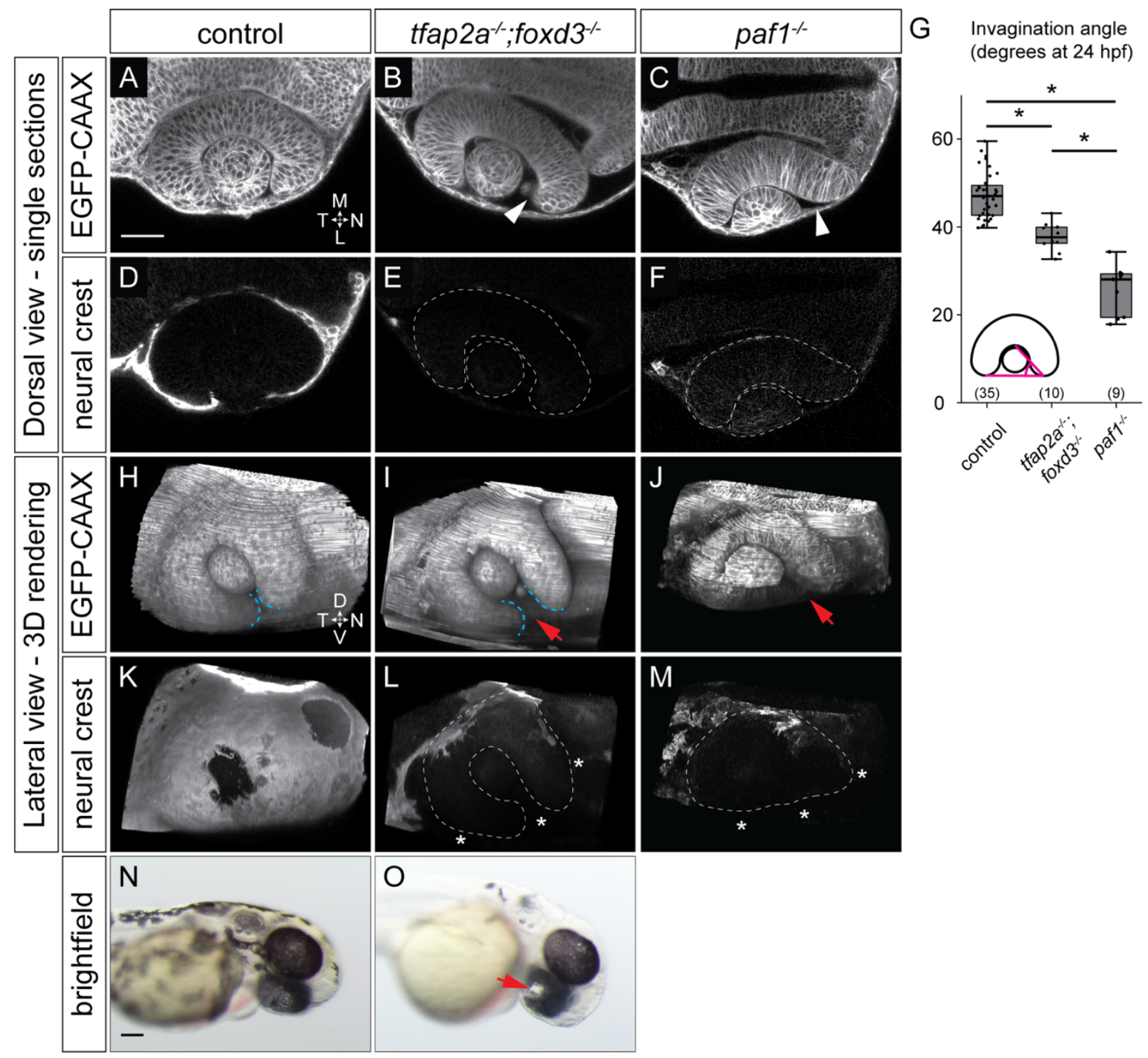

Figure 2. Optic cup morphogenesis is disrupted in neural crest mutants. (A-F) Dorsal view, single confocal sections of $24 \mathrm{hpf} T g(\operatorname{sox} 10$ :memRFP)-positive control (A, D), tfap2a;foxd3 double mutant (B, E), and pafl mutant (C, F) embryos. Sections shown are at the dorsal/ventral midpoint of the lens. EGFP-CAAX was used to visualize optic cup morphology: $(\mathrm{A}, \mathrm{B})$ $\operatorname{Tg}$ (bactin2:EGFP-CAAX); (C) injected with EGFP-CAAX mRNA. White dashed lines (E, F, L, $\mathrm{M})$ show optic cup boundaries. White arrowheads $(B, C)$ mark the nasal retina failing to fully enwrap the lens. $(\mathrm{G})$ Quantification of invagination angles, measured as shown in inset diagram. $* \mathrm{P}<0.001$ using Welch's t-test. (Control vs. paf1 mutant, $\mathrm{P}=6.59 \times 10$ - 7; control vs. tfap $2 a ;$ foxd3 double mutant, $\mathrm{P}=2.52 \times 10-7$; tfap2a;foxd3 double mutant vs. paf1 mutant, $\mathrm{P}=0.0001$.) Results are from 2-3 experiments; $n$ (embryos) shown at base of the graph. (H-M) Lateral view, 3D renderings of the embryos in (A-F). Blue dashed lines mark optic fissure margins. Note wideset optic fissure margins in the tfap2a;foxd3 mutant in (I); no discernable margins are visible in the pafl mutant at $24 \mathrm{hpf}$. White asterisks (L, M) indicate regions missing neural crest. $(\mathrm{N}, \mathrm{O})$

Brightfield images of $52 \mathrm{hpf}$ control and tfap $2 a ; f o x d 3$ double mutant embryos. Red arrows (I, J, O) indicate coloboma. Scale bars: $50 \mu \mathrm{m}$ in (A), $100 \mu \mathrm{m}$ in (N). M, medial; L, lateral; D, dorsal; $\mathrm{V}$, ventral; $\mathrm{N}$, nasal; T, temporal. 

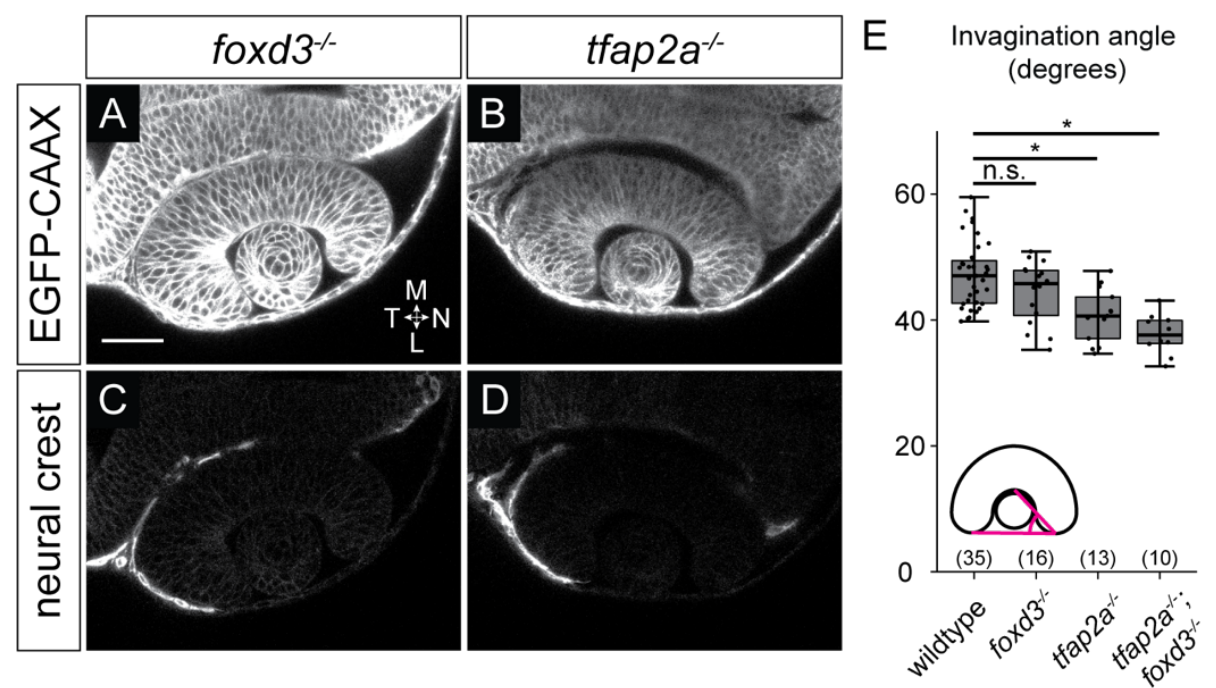

Figure S1. Invagination is disrupted in tfap 2 a but not foxd3 single mutants. (A-D) Dorsal view, single confocal sections of $24 \mathrm{hpf} T g$ (bactin2:EGFP-CAAX); $\operatorname{Tg}(\operatorname{sox} 10: m e m R F P)$ double transgenic foxd3 (A, C) and tfap2a (B, D) mutant embryos. Sections shown are at the dorsal/ventral midpoint of the lens. (E) Quantification of invagination angles, measured as shown in the inset diagram. $* \mathrm{P}<0.001$ using Welch's t-test. (Wildtype vs. tfap $2 a$;foxd 3 double mutant, $\mathrm{P}=2.52 \times 10^{-7}$; wildtype vs. tfap $2 a$ single mutant, $\mathrm{P}=0.0001$; wildtype vs. foxd 3 single mutant, $\mathrm{P}=0.07$.) Results are from 3 experiments; $\mathrm{n}$ (embryos) shown in the base of the graph. Scale bar, $50 \mu \mathrm{m}$. M, medial; L, lateral; N, nasal; T, temporal. 


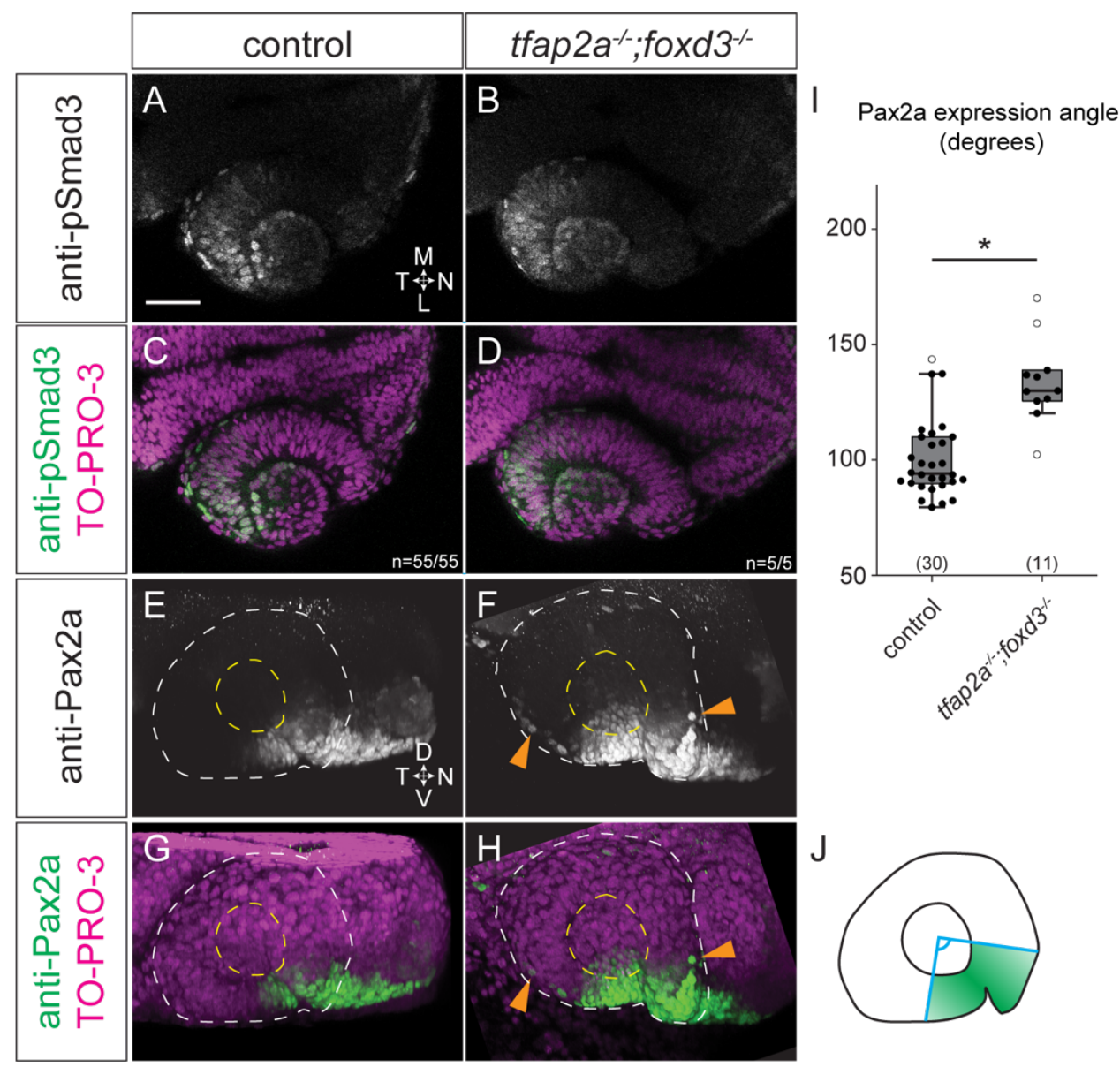

Figure 3. At 24 hpf, tfap2a;foxd3 double mutants display normal TGF-beta signaling, while Pax 2 a expression expands into the RPE. (A-D) Dorsal view, single confocal sections of 24 hpf control (A, C) and tfap2a;foxd3 double mutant (B,D) optic cups stained with anti-phosphoSmad3. Sections shown are at the dorsal/ventral lens midpoint. (E-H) Lateral view, 3D renderings of $24 \mathrm{hpf}$ control $(\mathrm{E}, \mathrm{G})$ and tfap $2 a$;foxd 3 double mutant $(\mathrm{F}, \mathrm{H})$ optic cups stained with anti-Pax2a. White dashed circles denote the boundary of the optic cup, yellow dashed circles display the boundary of the lens. Orange arrowheads in $(\mathrm{F}, \mathrm{H})$ indicate RPE cells which ectopically express Pax2a. (I) Angle measurements of the Pax2a expressing portion of control and tfap $2 a$;foxd3 double mutant optic cups. $* \mathrm{P}=5.39 \times 10^{-5}$ using Welch's t-test, white circles are outliers. Results are from 3 experiments; $n$ (embryos) shown in the base of the graph. Angles were measured from the lateral surface as diagrammed in $(\mathrm{J})$, with the vertex of the angle set at the center of the lens. Nuclei were counterstained with TO-PRO-3 (magenta); merges shown in (C, D, G, H). M, medial; L, lateral; D, dorsal; V, ventral; N, nasal; T, temporal. 


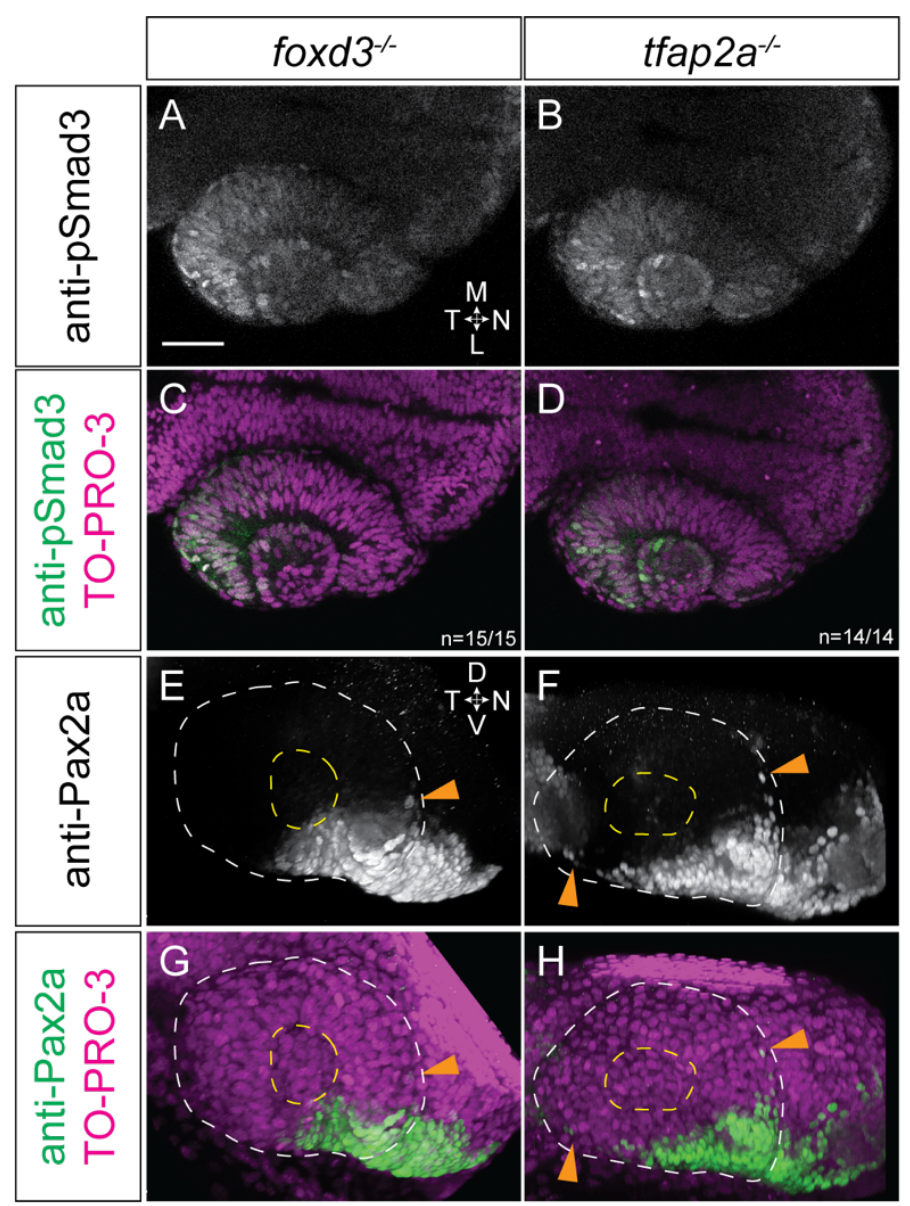

Figure S2. At 24 hpf, tfap $2 a$ and foxd 3 single mutants display normal TGF-beta signaling, while Pax2a expression expands into the RPE. (A-D) Dorsal view, single confocal sections of 24 hpf foxd3 mutant (A, C) and tfap2a mutant (B,D) optic cups stained with anti-phospho-Smad3.

Sections shown are at the dorsal/ventral lens midpoint. (E-H) Lateral view, 3D renderings of 24 hpf foxd 3 mutant $(\mathrm{E}, \mathrm{G})$ and tfap $2 a$ mutant $(\mathrm{F}, \mathrm{H})$ optic cups stained with anti-Pax $2 \mathrm{a}$. White dashed circles denote the boundary of the optic cup, yellow dashed circles display the boundary of the lens. Orange arrowheads in (E-H) indicate RPE cells which ectopically express Pax2a. Nuclei were counterstained with TO-PRO-3 (magenta); merges shown in (C, D, G, H). M, medial; L, lateral; D, dorsal; V, ventral; N, nasal; T, temporal. 

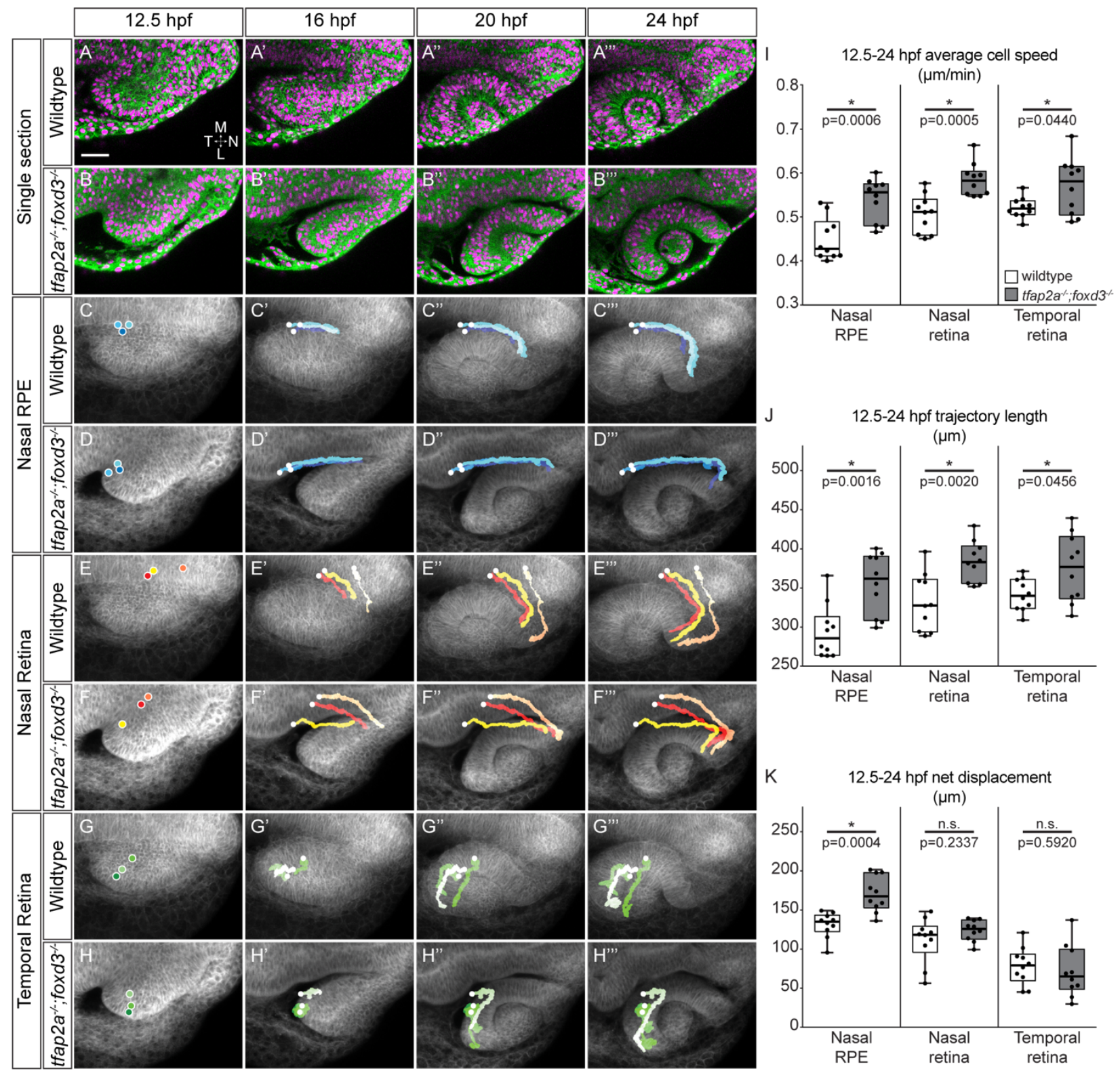

Figure 4. Cell movements throughout the optic cup are disrupted in tfap2a;foxd3 double mutants. Live imaging time series of optic cup morphogenesis from 12.5-24 hpf of Tg(bactin2:EGFP-CAAX) wildtype and tfap2a;foxd3 double mutant embryos. (A-B',') Dorsal view, single confocal sections from wildtype (A-A,',) and tfap 2a;foxd3 double mutant (B-B",') 4D datasets. EGFP-CAAX (green) labels cell membranes, while H2A.F/Z-mCherry (magenta) labels nuclei. (C-H',') Average projections of membrane (EGFP-CAAX) channel through 60 $\mu \mathrm{m}$ centered at the dorsal/ventral midpoint of the optic vesicle with indicated nuclear trajectories (nasal RPE, nasal retina, or temporal retina) overlaid. Trajectories were measured in 3D and generated by adding nuclear signal selections over time. (I-K) Average cell speed (I), total trajectory length $(\mathrm{J})$, and overall net displacement $(\mathrm{K})$ from cells which contribute to indicated region at 24 hpf. $n=10$ cells from each region ( 5 cells each from 2 embryos per genotype), backtracked from 24 to 12.5 hpf. P-values calculated using Welch's t-test. Scale bar, $50 \mu \mathrm{m}$. $\Delta \mathrm{T}$ between z-stacks, 2.75 minutes (wildtype) or 2.5 minutes (tfap2a;foxd3). M, medial; L, lateral; $\mathrm{N}$, nasal; $\mathrm{T}$, temporal. 


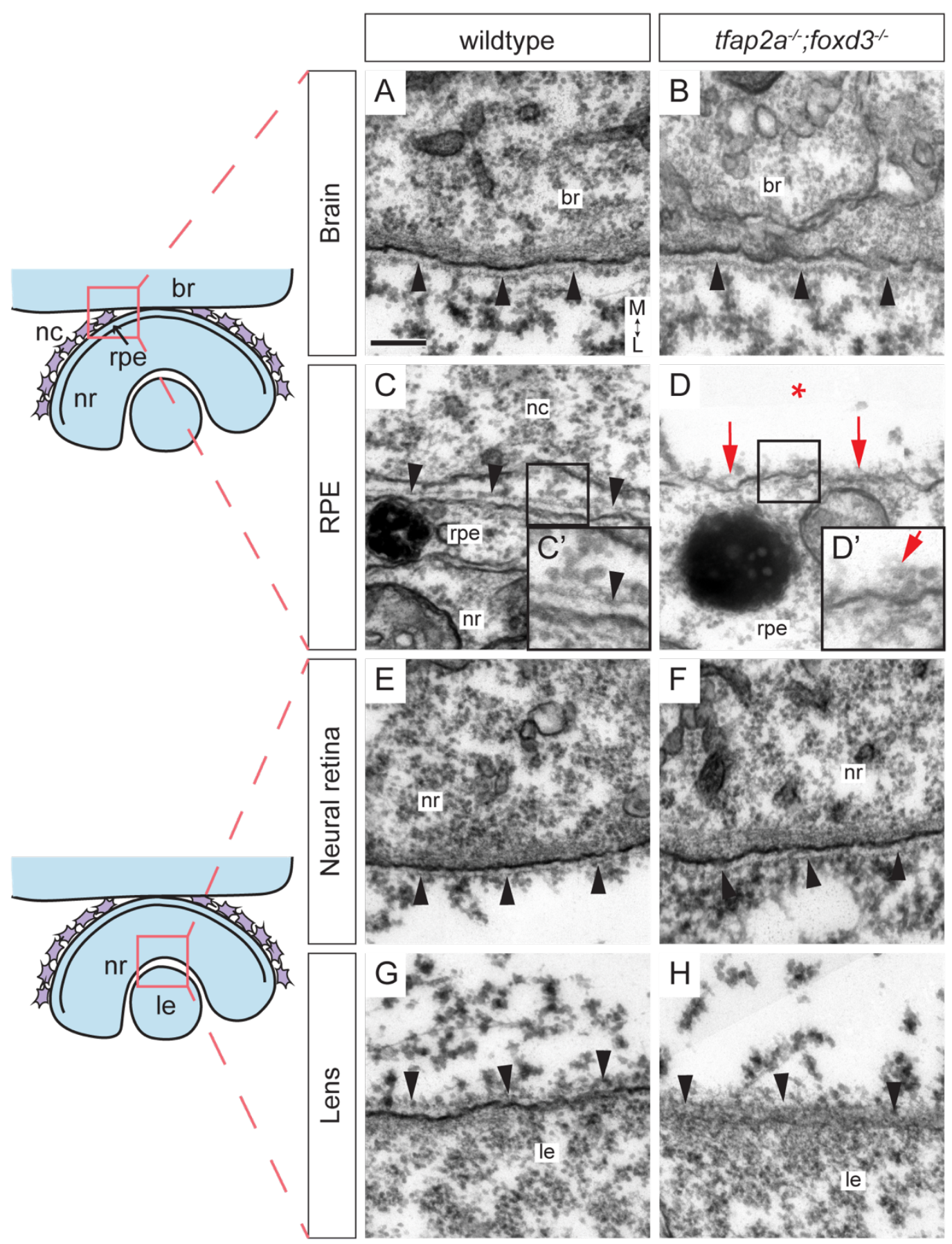

Figure 5. The basement membrane around the RPE is disrupted in tfap $2 a$;foxd3 double mutants. Transmission electron microscopy was used to visualize the basement membranes around the brain, RPE, neural retina, and lens in 24 hpf control (A, C, E, G) and tfap2a;foxd 3 double mutant $(\mathrm{B}, \mathrm{D}, \mathrm{F}, \mathrm{H})$ embryos, as diagrammed. The basement membrane around the RPE of tfap2a;foxd3 mutant embryos appears disorganized (D, D' red arrows) compared to wildtype (C, C'), while all other basement membranes appear normal (black arrowheads). Scale bar, $200 \mathrm{~nm}$. Magnification in $(A-H)=10,000 x,\left(C^{\prime}, D^{\prime}\right)=20,000 x$. br, brain; nc, neural crest cell; nr, neural retina; rpe, retinal pigment epithelium; le, lens. All images are transverse sections, anterior views. M, medial; L, lateral. 


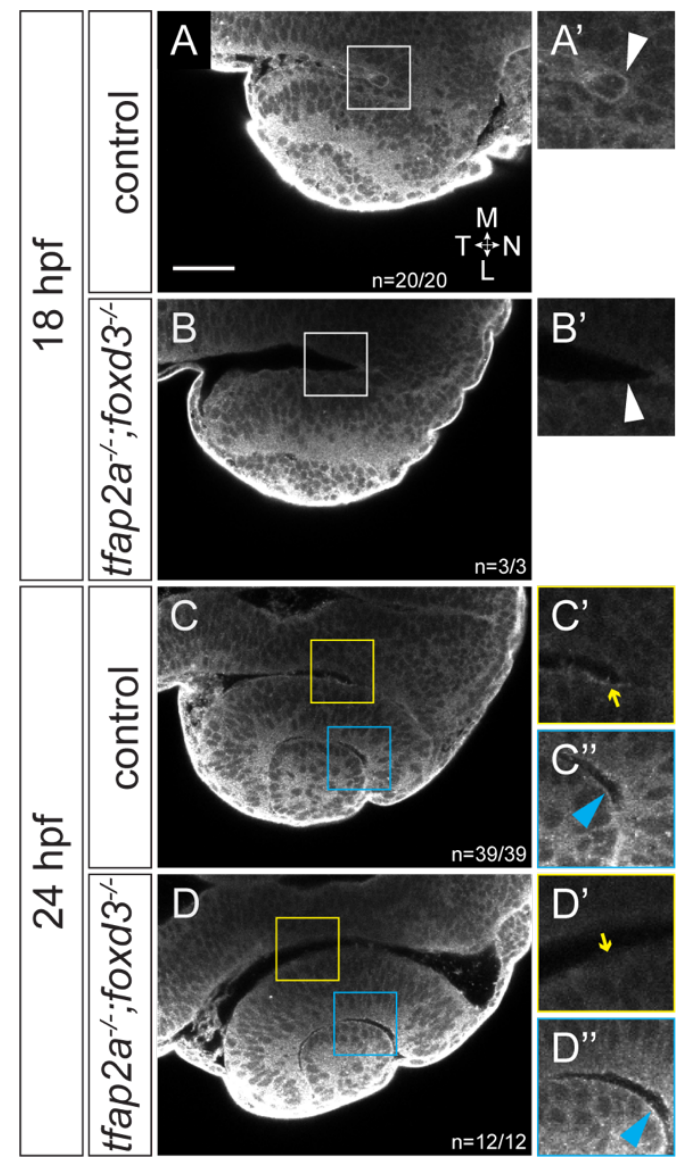

Figure 6. Nidogen protein is absent from the RPE side of the optic cup in tfap2a;foxd 3 double mutants. At $18 \mathrm{hpf}$, nidogen protein is detected by immunofluorescence in neural crest cells between the brain and developing RPE in the optic stalk furrow (A, magnified in A'). This expression is missing in tfap2a;foxd3 double mutant embryos at $18 \mathrm{hpf}$ and there are no cells visible in the same space (B, magnified in B'). At 24 hpf in control embryos, a nidogen ECM surface is detectable on along both the RPE (yellow box in C, yellow arrow in C') and at the lens-retina interface (C, C', blue arrowhead). In $24 \mathrm{hpf}$ tfap $2 a$;foxd 3 double mutants, nidogen is not detectable along the RPE (D', yellow arrow), but is still present in the ECMs at the lensretina interface (D', blue arrowhead). Dorsal view, single confocal sections. Scale bar, $50 \mu \mathrm{m}$. 


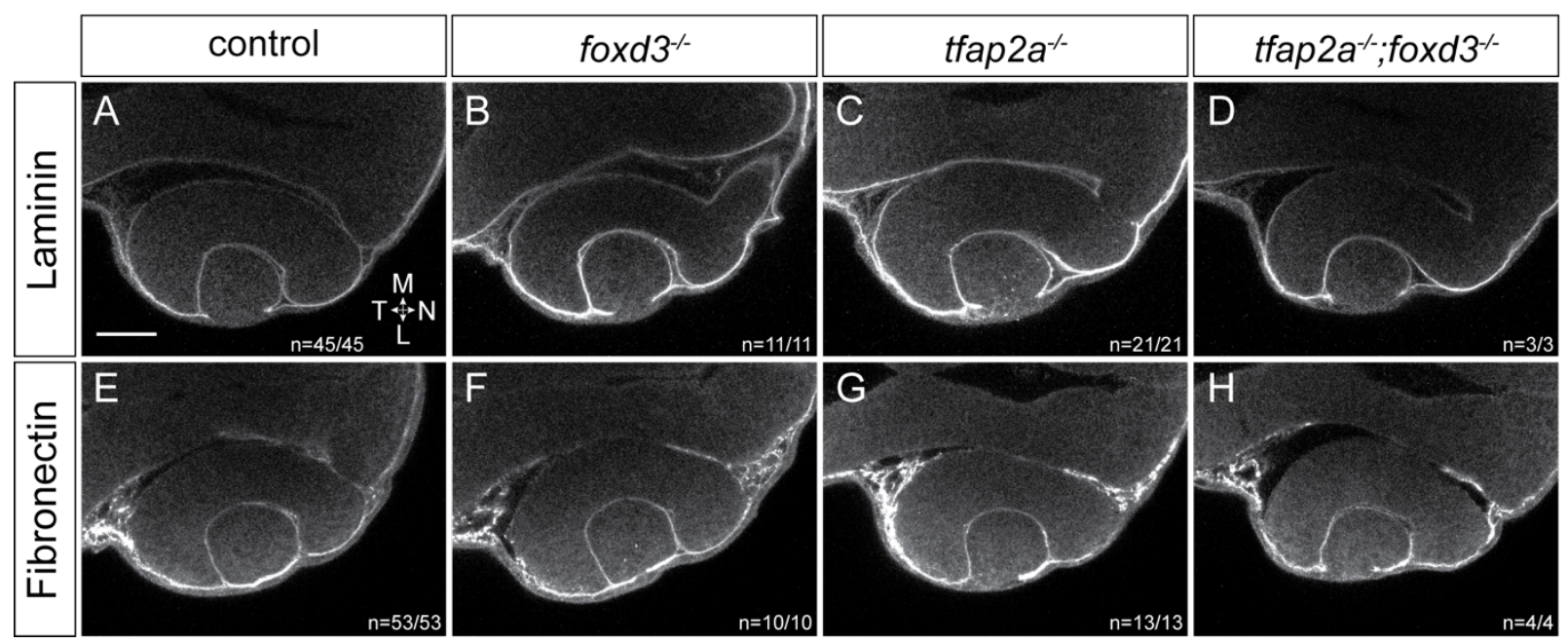

Figure S3. Laminin and fibronectin localization are unaffected in tfap $2 a$;foxd 3 double mutants. At $24 \mathrm{hpf}$, both laminin (A-D) and fibronectin (E-H) are found around the developing optic cup in all genotypes shown. Dorsal view, single confocal sections. Scale bar, $50 \mu \mathrm{m}$. M, medial; L, lateral; N, nasal; T, temporal. 


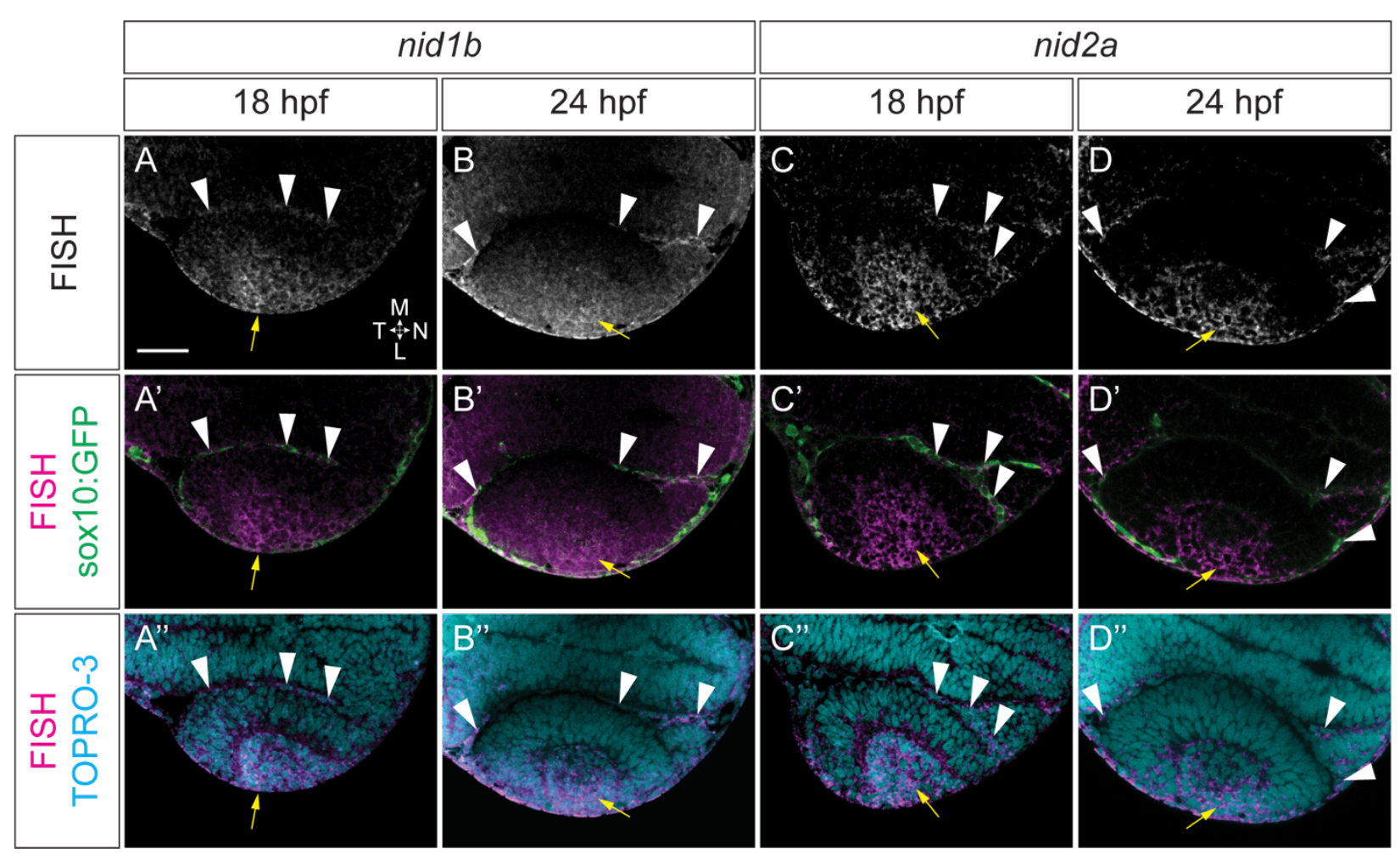

Figure 7. Nidogen $1 \mathrm{~b}$ and $2 \mathrm{a}$ are expressed in the neural crest and developing lens. In situs were performed in $\operatorname{Tg}(\operatorname{sox} 10: G F P)$ embryos at 18 (A-A', C-C' ') and $24 \mathrm{hpf}(\mathrm{B}-\mathrm{B}$ ', D-D' '). (A-D) Fluorescence in situ hybridization with probes against nidlb (A,B) and nid2a (C,D). (A'-D') FISH merged with sox10:GFP expression (green) to visualize colocalization between FISH and $\mathrm{GFP}^{+}$neural crest cells (white arrowheads). GFP signal was amplified after hybridization using an anti-GFP antibody. (A'-D') FISH merged with nuclei counterstained with TO-PRO-3.

Yellow arrows denote lens expression in each case. Dorsal view, single confocal sections. Scale bar, $50 \mu \mathrm{m}$. 


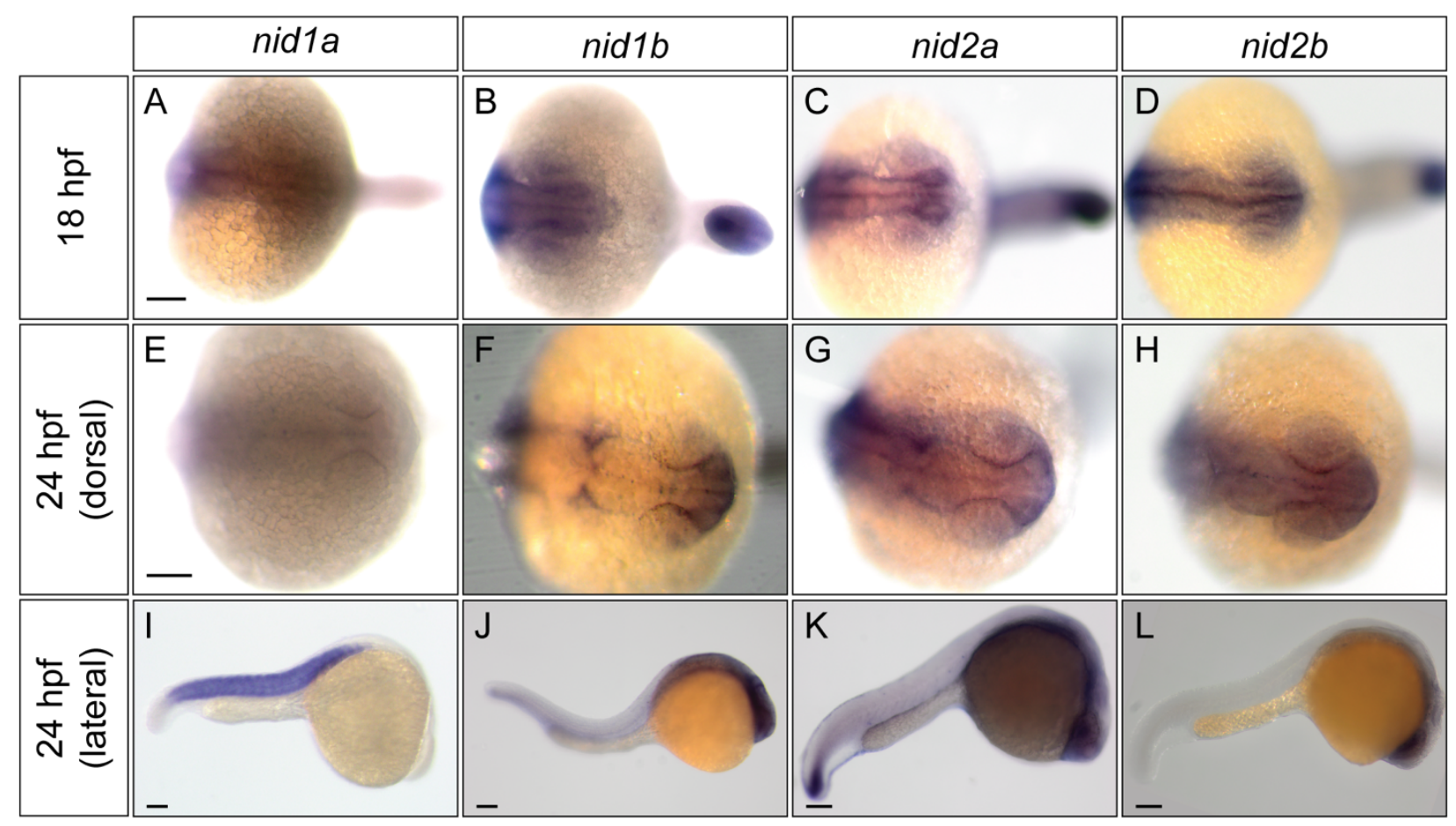

Figure S4. Zebrafish nidogen mRNA expression patterns at 18 and $24 \mathrm{hpf}$. Scale bars, $100 \mu \mathrm{m}$. 


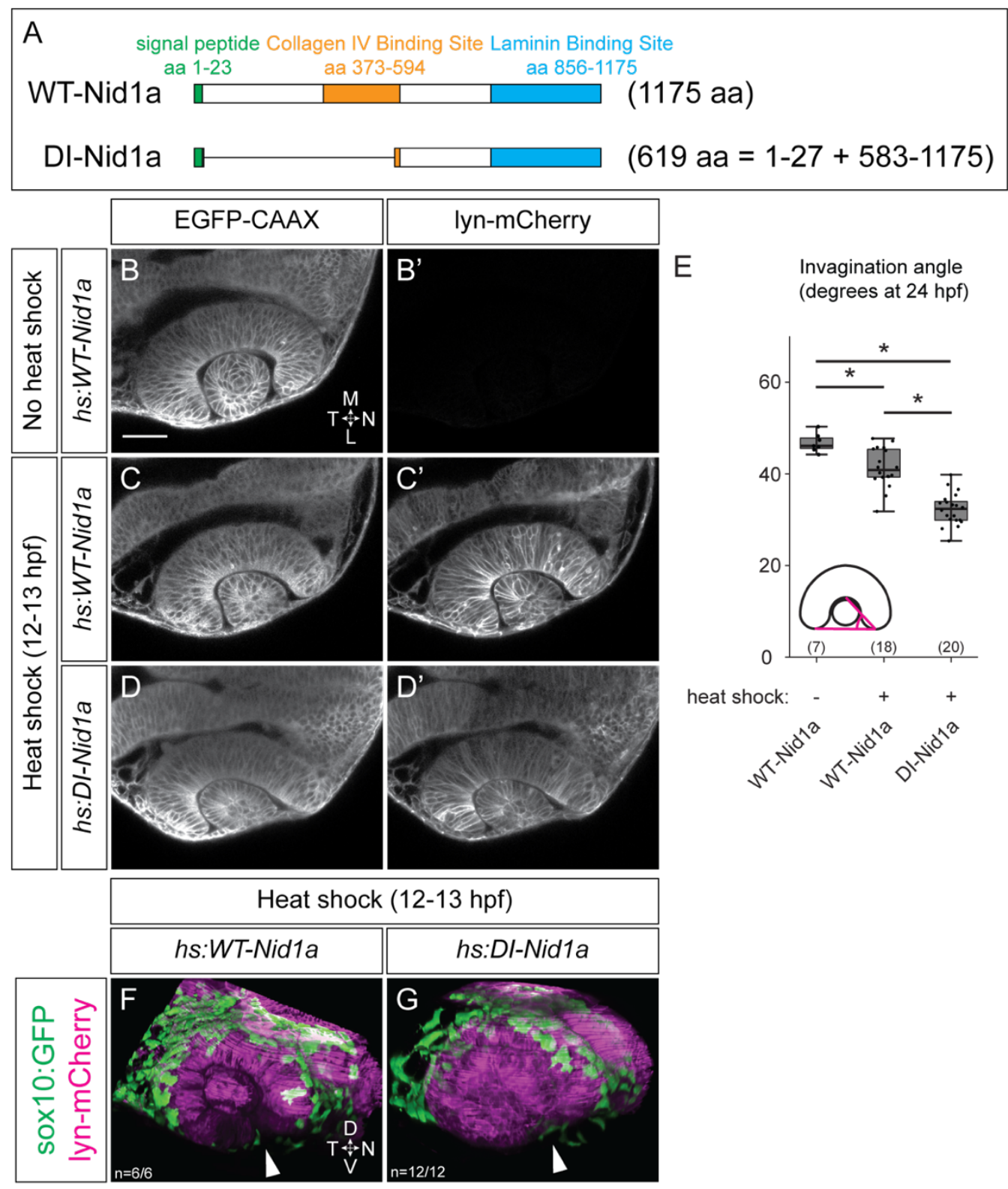

Figure 8. Dominant-interfering nidogen disrupts optic cup morphogenesis. (A) Schematics of full length (WT) and dominant-interfering (DI) Nidogen 1a. (B-D') Dorsal view, single confocal sections. $T g$ (bactin2:EGFP-CAAX) females were crossed to either $h s: W T-N i d 1 a$ (B-C') or $h s: D I-N i d l a(\mathrm{D}, \mathrm{D}$ ') transgenic males. Control embryos (B, B') were not heat shocked, show no lyn-mCherry expression; experimental embryos were heat shocked 12-13 hpf (C-D'). (E) Quantification of invagination angles, measured as shown in inset diagram. ${ }^{*} \mathrm{P}<0.005$ using Welch's t-test. (WT-Nidla no heat shock vs. heat shock, $\mathrm{P}=0.001 ;$ WT-Nidla heat shock vs. DINidla heat shock, $\mathrm{P}=4.34 \times 10^{-10}$; WT-Nidla no heat shock vs. DI-Nidla heat shock, $\mathrm{P}=7.76 \times 10^{-}$ 11; DI-Nidla no heat shock vs. heat shock (not shown), $\mathrm{P}=6.46 \times 10^{-14}$.) Results are from 3 experiments; $\mathrm{n}$ (embryos) shown in base of graph. (F, G) Lateral view, 3D renderings at 24 hpf. sox 10:GFP transgenic females were crossed to either $h s: W T-N i d l a(\mathrm{~F})$ or $h s: D I-N i d l a(\mathrm{G})$ transgenic males, embryos heat shocked 12-13 hpf. GFP-positive neural crest cells migrate around the optic cup and into the optic fissure (white arrowheads) in both conditions. Scale bar, $50 \mu \mathrm{m} . \mathrm{M}$, medial; L, lateral; N, nasal; T, temporal. 


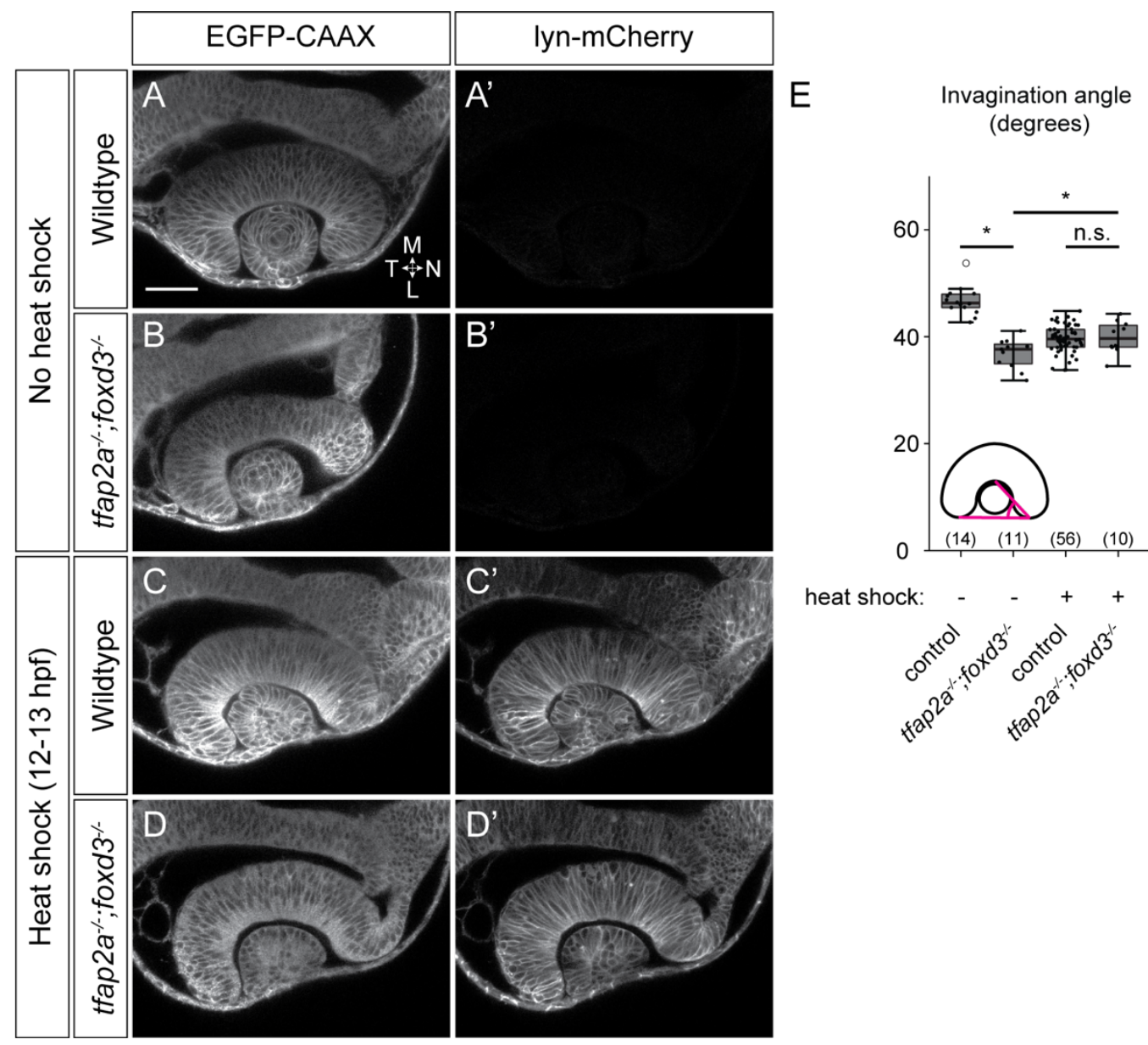

Figure 9. Overexpression of Nidla partially rescues optic cup morphogenesis in tfap $2 a$;foxd3 double mutants. Dorsal view, single confocal sections from $24 \mathrm{hpf} T g(h s: W T$-Nidla $)$-positive embryos.(A-B') EGFP-CAAX and lyn-mCherry channels from wildtype (A, A') and tfap2a;foxd3 double mutant (B, B') embryos which were not subjected to a heat shock. (C-D') EGFP-CAAX and lyn-mCherry channels from wildtype (C, C') and tfap2a;foxd3 double mutant (D, D') embryos which were heat shocked from 12-13 hpf. (E) Quantification of invagination angles, measured as shown in the inset diagram. ${ }^{*} \mathrm{P}<0.05$ using Welch's t-test; n.s., not significant. (Wildtype (no heat shock) vs. tfap $2 a$;foxd3 double mutant (no heat shock), $\mathrm{P}=1.42 \times 10^{-8}$; tfap $2 a$;foxd 3 double mutant no heat shock vs. heat shock, $\mathrm{P}=0.03$; wildtype (heat shock) vs. tfap2a;foxd3 (heat shock), $\mathrm{P}=0.76$.) Results are from 3 experiments; $\mathrm{n}$ (embryos) shown in the base of the graph. Scale bar, $50 \mu \mathrm{m}$. M, medial; L, lateral; N, nasal; T, temporal. 


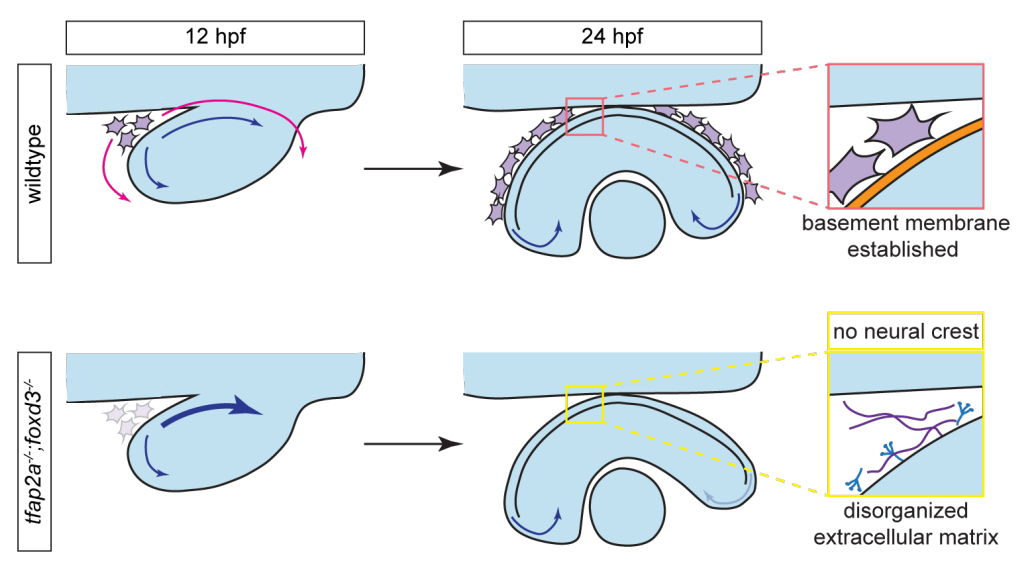

Figure 10. Model of neural crest function during optic cup morphogenesis. Optic cup morphogenesis in a wildtype embryo (top row) and a tfap $2 a$;foxd3 double mutant (bottom row). In wildtype embryos, neural crest cells (purple) begin to migrate around the optic vesicle (magenta arrows) starting around $12 \mathrm{hpf}$ and enwrap the retinal pigment epithelium by $24 \mathrm{hpf}$. During this process neural crest cells express and deposit nidogen, which establishes the basement membrane (orange) around the RPE and restricts cell movements within the prospective eye (blue arrows), in turn enabling proper rim movement and optic cup morphogenesis. In tfap2a;foxd 3 double mutants which lack most neural crest cells, the ECM surrounding the RPE is disorganized and does not form a coherent basement membrane. Cell movements within the optic vesicle are unrestricted (heavy blue arrow), leading to the abnormal position of the anterior RPE and disrupted rim movement of cells which would normally migrate into the neural retina. 
Supplemental Movie 1. Neural crest migration during optic cup morphogenesis. 12.5-24.5 hpf timelapse of a $\operatorname{Tg}(\beta$-actin2:EGFP-CAAX); $\operatorname{Tg}(\operatorname{sox} 10: m e m R F P)$ double transgenic embryo. EGFP-CAAX labels all cell membranes (green), while membrane-bound RFP (magenta) labels only the neural crest. Dorsal view, single confocal section through the dorsal/ventral midpoint of the optic cup. Nasal (anterior) is to the right, temporal (posterior) to the left. $\Delta \mathrm{T}$ between zstacks, 3 minutes 30 seconds.

Supplemental Movie 2. Neural crest migration during optic cup morphogenesis. 12.5-24.5 hpf timelapse of a $\operatorname{Tg}(\beta$-actin2:EGFP-CAAX);Tg(sox 10:memRFP) double transgenic embryo. Lateral view, 3D rendering of the same timelapse dataset shown in Supplemental Movie 1. Only the RFP channel is shown (grayscale) to enable visualization of neural crest cell migration. Nasal (anterior) is to the right, temporal (posterior) to the left, dorsal to the top, ventral to the bottom. $\Delta \mathrm{T}$ between z-stacks, 3 minutes 30 seconds

Supplemental Movie 3. Nuclear trajectories visualized in in three dimensions. 3D rendered rotation of $24 \mathrm{hpf}$ timepoints showing representative trajectories of nuclei from cells in the nasal retina (orange), nasal RPE (blue) and temporal retina (green). Membrane channel is displayed in grayscale.

Supplemental Movie 4. Wildtype nasal RPE nuclear trajectories from 12.5-24 hpf. Representative trajectories over membrane channel average (grayscale). $\Delta \mathrm{T}$ between z-stacks, 2 minutes 45 seconds.

Supplemental Movie 5. tfap2a;foxd3 double mutant nasal RPE nuclear trajectories from 12.5-24 hpf. Representative trajectories over membrane channel average (grayscale). $\Delta \mathrm{T}$ between $\mathrm{z}-$ stacks, 2 minutes 30 seconds.

Supplemental Movie 6. Wildtype nasal retina nuclear trajectories from 12.5-24 hpf. Representative trajectories over membrane channel average (grayscale). $\Delta \mathrm{T}$ between $\mathrm{z}$-stacks, 2 minutes 45 seconds.

Supplemental Movie 7. tfap2a;foxd3 double mutant nasal retina nuclear trajectories from 12.5$24 \mathrm{hpf}$. Representative trajectories over membrane channel average (grayscale). $\Delta \mathrm{T}$ between $\mathrm{z}-$ stacks, 2 minutes 30 seconds.

Supplemental Movie 8. Wildtype temporal retina nuclear trajectories from 12.5-24 hpf. Representative trajectories over membrane channel average (grayscale). $\Delta \mathrm{T}$ between $\mathrm{z}$-stacks, 2 minutes 45 seconds.

Supplemental Movie 9. $t$ fap $2 a$; foxd 3 double mutant temporal retina nuclear trajectories from 12.5-24 hpf. Representative trajectories over membrane channel average (grayscale). $\Delta \mathrm{T}$ between z-stacks, 2 minutes 30 seconds. 